\title{
HST/WFPC2 morphologies and color maps of distant luminous infrared galaxies ${ }^{\star}$
}

\author{
X. Z. Zheng ${ }^{1}$, F. Hammer ${ }^{1}$, H. Flores ${ }^{1}$, F. Assémat ${ }^{1}$, and D. Pelat ${ }^{2}$ \\ 1 GEPI, Observatoire de Paris-Meudon, 92195 Meudon, France \\ ${ }^{2}$ LUTH, Observatoire de Paris-Meudon, 92195 Meudon, France
}

Received 2 December 2003 / Accepted 17 March 2004

\begin{abstract}
Using HST/WFPC2 imaging in $F 606 \mathrm{~W}$ (or $F 450 \mathrm{~W}$ ) and $F 814 \mathrm{~W}$ filters, we obtained the color maps in observed frame for 36 distant $(0.4<z<1.2)$ luminous infrared galaxies (LIRGs, $L_{\mathrm{IR}}(8-1000 \mu \mathrm{m}) \geq 10^{11} L_{\odot}$ ), with average star formation rates of $\sim 100 M_{\odot} \mathrm{yr}^{-1}$. Stars and compact sources are taken as references to align images after correction of geometric distortion. This leads to an alignment accuracy of 0.15 pixel, which is a prerequisite for studying the detailed color properties of galaxies with complex morphologies. A new method is developed to quantify the reliability of each pixel in the color map without any bias against very red or blue color regions.

Based on analyses of two-dimensional structure and spatially resolved color distribution, we carried out morphological classification for LIRGs. About $36 \%$ of the LIRGs were classified as disk galaxies and $22 \%$ as irregulars. Only $6(17 \%)$ systems are obvious ongoing major mergers. An upper limit of $58 \%$ was found for the fraction of mergers in LIRGs with all the possible merging/interacting systems included. Strikingly, the fraction of compact sources is as high as $25 \%$, similar to that found in optically selected samples. From their $K$ band luminosities, LIRGs are relatively massive systems, with an average stellar mass of about $1.1 \times 10^{11} M_{\odot}$. They are related to the formation of massive and large disks, from their morphologies and also from the fact that they represent a significant fraction of distant disks selected by their sizes. If sustained at such large rates, their star formation can double their stellar masses in less than 1 Gyr. The compact LIRGs show blue cores, which could be associated with the formation of the central region of these galaxies. We find that all LIRGs are distributed along a sequence which relate their central color to their concentration index. This sequence links compact objects with blue central color to extended ones with relatively red central color, which are closer to the local disks. We suggest that there are many massive disks which have been forming a large fraction of their stellar mass since $z=1$. For most of them, their central parts (bulge?) were formed prior to the formation of their disks.
\end{abstract}

Key words. galaxies: formation - galaxies: evolution - infrared: galaxies

\section{Introduction}

The evolution of the cosmic star formation density (CSFD) shows the history of the stellar mass assembly averaged over all galaxies. A sharp decline of the CSFD since $z \sim 1$ has been found, whereas large uncertainties still remain at higher redshifts, particularly due to the uncertainties and biases regarding dust extinction (e.g. Madau et al. 1996; Hammer et al. 1997). Investigations of global stellar mass density as a function of redshift indicate that more than one quarter, probably up to half of the present day stars were formed since $z \sim 1$ (Dickinson et al. 2003, and references therein). This is in agreement with an integration of the CSFD if the latter accounts for all the light re-radiated at IR wavelengths (Flores et al. 1999). Hence the star-forming activities since $z \sim 1$ still play an important role in

Send offprint requests to: $\mathrm{X}$. Z. Zheng, e-mail: Xianzhong.Zheng@obspm.fr

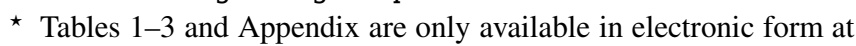
http://www. edpsciences.org the formation of the galaxy Hubble sequence seen in the local universe.

Hubble Space Telescope (HST) observations show that the merger rate increases significantly at $z \sim 1$, compared with that in the local universe (Le Fèvre et al. 2000; Conselice et al. 2003). Such events were claimed to be related to dwarf galaxies while massive systems formed before redshift 1 (Brinchmann \& Ellis 2000; Lilly et al. 1998; Schade et al. 1999). However, with Infrared Space Observatory (ISO) mid-infrared imaging, Flores et al. (1999) inferred that a substantial fraction of star formation since $z \sim 1$ is associated with the LIRGs. These objects are luminous star-forming galaxies at intermediate redshifts $(z \sim 0.5$ to 1$)$, different from the faint blue galaxy population (Genzel \& Cesarsky 2000; Franceschini et al. 2003). It is widely accepted that merger/interaction is very efficient in pushing gas into the nuclear region and triggering violent star formation. Therefore LIRGs are suspected to be merging systems and the evolution of these galaxies is linked to the decline of the merger rate (Elbaz et al. 2002). Although HST imaging 
showed that most of the LIRGs are luminous disk/interacting galaxies (Flores et al. 1999), systematic investigation of their properties is still required to understand their formation and evolution, as well as to link them to counterparts in the local universe.

Morphological classification is essential to reveal the nature of distant LIRGs. However, at high redshifts, it becomes difficult to classify galaxy morphology securely because the images of the high- $z$ galaxies suffer from reduced resolution, band-shifting and cosmological surface brightness dimming effects, compared with the local objects. With the HST Wide Field Planet Camera 2 (WFPC2), high resolution imaging in two or more bands with spatially resolved color distribution can be used to investigate the distribution of the stellar population, which is complementary to addressing the appearance in a single band. Furthermore, the star-forming regions and dusty regions can stand out in the color map. This is very important to the study of LIRGs, in which these regions are expected to be numerous.

The Canada-France Redshift Survey (CFRS) fields are among the most studied fields at various wavelengths. Two CFRS fields $0300+00$ and $1415+52$ had been observed deeply by ISOCAM at $15 \mu \mathrm{m}$ (Flores et al. 1999, 2004) and by HST (Brinchmann et al. 1998). To perform detailed analyses of morphology, photometry and color distribution for distant LIRGs, additional HST images through blue and red filters have been taken to complement the color information of the two CFRS fields (PI: Hammer, Prop. 9149). In this work, we present the preliminary results of the color distribution of distant LIRGs. We correct additional effects in HST images and recenter them accurately, which allows us to access the color maps of complex galaxies. We also implement a method to quantify the signal-to-noise $(S / N)$ ratio of the color image to give a reasonable cut for the target area in color maps.

This paper is organized as follows. Section 2 describes the HST imaging observations and the archive data we adopt. In Sect. 3, we describe the various effects which have to be corrected in aligning images in different WFPC2 filters. In Sect. 4 , we describe the method we use to generate the color maps. In Sect. 5, we summarize the morphological properties of the distant LIRGs. The results we obtained of the LIRGs are discussed in Sect. 6. Brief conclusions are given in Sect. 7. Throughout this paper we adopt $H_{0}=70 \mathrm{~km} \mathrm{~s}^{-1} \mathrm{Mpc}^{-1}, \Omega_{\mathrm{M}}=0.3$ and $\Omega_{\Lambda}=$ 0.7. Unless specified, we exclude the PC chip and the unit of pixel refers to that in WF chips. The bands $B_{450}, V_{606}$ and $I_{814}$ refer to HST filters $F 450 W, F 606 W$ and $F 814 W$, respectively. The Vega system is adopted for our photometry.

\section{Observations}

Ground-based spectroscopic redshift identification in the CFRS was carried out with CFHT telescope for objects brighter than $22.5 \mathrm{mag}\left(I_{\mathrm{AB}}\right)$ in five $10^{\prime} \times 10^{\prime}$ fields (see Crampton et al. 1995 for details). With improved data reduction, Flores et al. (2004) present the updated catalogs of the deep ISOCAM observations at $15 \mu \mathrm{m}$ for the two CFRS fields $0300+00$ and $1415+52$ (see also Flores et al. 1999).
Using high resolution HST/WFPC2 imaging, the distant galaxies up to $z \sim 1$ can be spatially resolved. Three HST fields were observed in the $F 606 \mathrm{~W}$ and $F 814 \mathrm{~W}$ filters in the two CFRS fields with ISOCAM observations (in Cycle 10, PI: Hammer, Prop. 9149), and two fields in the $F 606 W$ filter were observed to complement the observations in $F 814 \mathrm{~W}$ during Cycle 8 (PI: Lilly, Prop. 8162). The F606W and F814W filters correspond to rest-frame $U(3634 \AA)$ and $V(4856 \AA)$ at a redshift 0.65 . Those fields were chosen to maximize the number of LIRGs contained in each field. We also collected the HST imaging data with two band observations in the CFRS fields. A detailed description of the previous CFRS field HST imaging survey was presented in Brinchmann et al. (1998). The CFRS 1415+52 field partially overlaps the Groth Strip Survey (GSS, Groth et al. 1994). We included the GSS imaging data in our analysis.

Table 1 summarizes the HST imaging data used in this analysis. The total exposure time is usually more than $6000 \mathrm{~s}$, in which the surface brightness corresponding to $1 \sigma$ above the background is $\sim 25.5 \mathrm{mag} \operatorname{arcsec}^{-2}$. Note that the GSS is relatively shallow except for one very deep field. Here we list the seven of 28 GSS fields covering the CFRS 1415+52 field. The 28 GSS fields are composed of 27 fields observed in Prop. 5090 (PI: Groth) and one very deep field in Prop. 5109 (PI: West Phal). Simard et al. (2002) carried out a detailed morphological analysis on the GSS observations. They also provided the physical scale and absolute magnitude for objects with spectroscopic redshift identification using the Keck telescope. We quoted these results directly and further details can be found in their paper. The observation was divided into $N$ exposures (Cols. 4 and 8 in Table 1) aimed at removing cosmicrays and correcting the bad/hot pixels.

\section{Image alignment}

Studies have been reported investigating color maps for spheroidal galaxies at intermediate redshifts (e.g. Abraham et al. 1999; Ellis et al. 2001). In generating the color map, a difficulty is to align two images accurately so as to keep each pixel of the same object at the same position in two images. For the spheroidal galaxies, generally the brightness peaks at the galaxy center in different bands. It would be technically easy to correct relative shifts between two images if the galaxy center is used as a reference. For the galaxies with complex morphologies, however, caution should be applied in determining the relative shifts because their irregular morphologies as well as contamination from star-forming regions could easily effect efforts to find a reliable reference point (e.g. the galaxy center in spheroidal galaxies). Such a difficulty becomes more serious for galaxies at intermediate redshifts when band-shifting effects become significant. The uncertainty in aligning two images is required to be much smaller than one pixel in addressing color distribution pixel-by-pixel. We model the color map $V_{606}-I_{814}$ to imitate the effect of the alignment offset on the color map. We use the modeled HST/WFPC2 images of an elliptical galaxy at redshift 0.299 with a de Vaucouleurs bulge+exponential disk structure $(B / T=$ 0.84) given by GIM2D (Simard et al. 2002), to avoid 

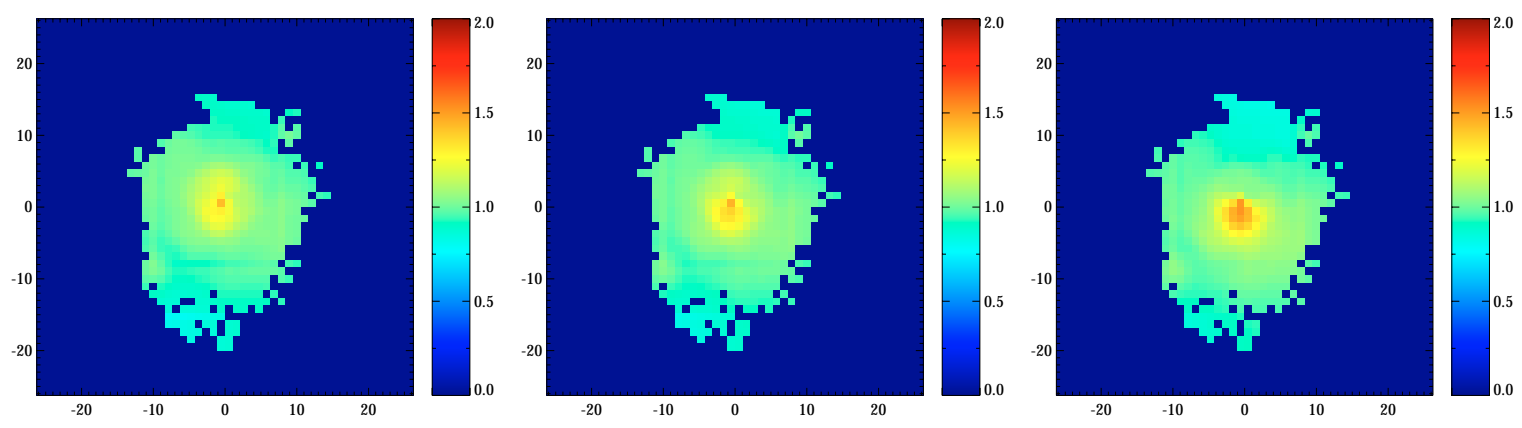

Fig. 1. Imitated HST/WFPC2 $V_{606}-I_{814}$ color maps for an elliptical galaxy at redshift 0.299 when the offsets between blue and red images are 0.0 pixel (left panel), 0.15 pixel (middle panel) and 0.30 pixel (right panel). The color maps are $50 \times 50$ pixel. (See the electronic edition for a color version of this figure.)

contamination by the intrinsic color fluctuation. Figure 1 illustrates that an offset as small as $\sim 0.3$ pixel will cause false structure "half-blue and half-red" in the color map (right panel), compared with the color map with zero offset (left panel). Such an effect becomes marginal when the offset decreases to 0.15 pixel (middle panel), which is the typical uncertainty in our image alignment. Instead of operating on individual objects, we dealt with the whole images in data processing. Our method of data reduction to align images is summarized below.

The raw HST images were processed using the standard STScI pipeline. For HST/WFPC2 observations in integerpixel dither mode with telescope relative offsets larger than a few pixels, camera geometric distortion will cause an additional shift (increasing toward the CCD corners, see HST/WFPC2 handbook for more details). We correct the geometric distortion for each individual exposure in a data set before combining them. Table 1 tabulates the dither offsets, usually twenty pixels (in which an additional shift of about half a pixel due to the geometric distortion is present at the WF chip corners) except for those in Proposal 5449 and GSS, in which consecutive exposures were taken at the same position and the distortion correction was not applied. The shifts between the exposures were obtained using two approaches, cross-correlation and point source reference. The cross correlation technique is to shift and/or rotate one image relative the other to maximize the cross-correlation between the two images, i.e. the best match. In the later approach, the shifts are derived from a comparison of the locations of a number of point and point-like sources in individual exposures. In our work, the point and point-like sources refer to the objects satisfying Full Width at Half Magnitude $(F W H M)<2.5$ pixels, $17<m_{814}<22,17<m_{450}<23$ and $17<m_{606}<23$. These criteria exclude the extended objects and those saturated or faint. For each WFPC2 field, at least three reference sources are used to derive the relative shifts between the different exposures. Both approaches use real images/objects to derive the shifts and hence are free from guide star acquisition uncertainties. In general, the derived shifts are remarkably consistent with each other within 0.08 pixel and even better for crowded fields. For sparse fields and some fields with large dither offsets, the cross-correlation is not the best approach and measurement using the point source reference will be adopted. The cross-correlation is also not suitable to find the rotation angle and the shifts between images in different bands. The morphologies and brightness of the astronomical sources may differ in one wavelength window from those in another. The crosscorrelation would be biased by sources with a real center offset between different bands, which is often seen in spiral and irregular galaxies. Geometric distortion correction, cosmic-ray removement and image combination are accomplished using the STSDAS/DITHER package (version 2.0, Koekemoer et al. 1995).

In some of the fields, blue images and red images were taken in different cycles, i.e. the HST telescope pointings in two bands were not the same, which results in different relative rotations and shifts between them. We have used pointlike sources as references to determine the rotations and the shifts. In practice, we obtain the relative rotation angle from astrometric information recorded in the image header. The keyword ORIENTAT provides the position angle of the telescope pointing. Normally the uncertainty of the position angle determination is 0.003 degree, compared to a rotation deviation of 0.01 degree causing a 0.1 pixel offset at the corners of a WF chip. Table 2 lists the shift and the rotation angle of the blue image relative to the red one for the WF3 chip. Note that in image combination, the first image of the data set is always taken as the reference to stack images.

To show the advantages of the point source reference method, a systematic shift exists between the $V_{606}$ image and $I_{814}$ image in the observations of the 27 GSS fields in Prop. 5090. The observation of each field was split into 8 exposures in $V_{606}$ and $I_{814}$ bands alternately at the same location, i.e. no offset between consecutive exposures and 4 exposures for each filter. For the 8 exposures of each field, we derive the shifts relative to the first $I_{814}$ exposure by comparing the positions of point and point-like sources in individual exposures. It is assumed that there is no relative shift/rotation between individual chips during each exposure. At least 3 reference sources (usually 7 , or more than 10 for some fields) in three WF chips are used to give median shifts in $X$ and $Y$ axes. Figure 2 illustrates the distribution of the relative shifts of the remaining 7 exposures to the first one for the 27 GSS fields. It reveals the systematic offset between $V_{606}$ and $I_{814}$ images. The shifts for each field are tabulated in Table 2. Combining the 27 fields, we get a median shift of $\Delta x=0.20$ pixel and $\Delta y=0.08$ pixel. 


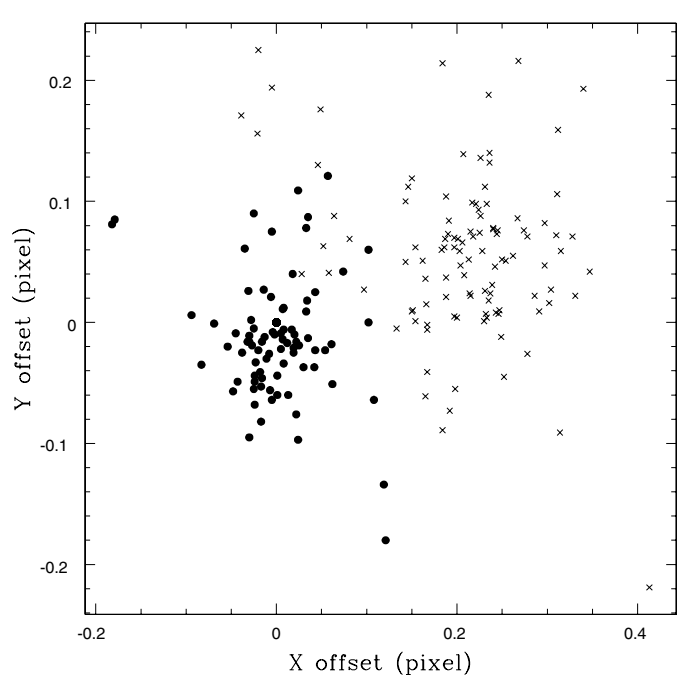

Fig. 2. Systematic offsets between $V_{606}$ and $I_{814}$ images in 27 GSS fields. Solid circle is $I_{814}$ image and cross is $V_{606}$ image.

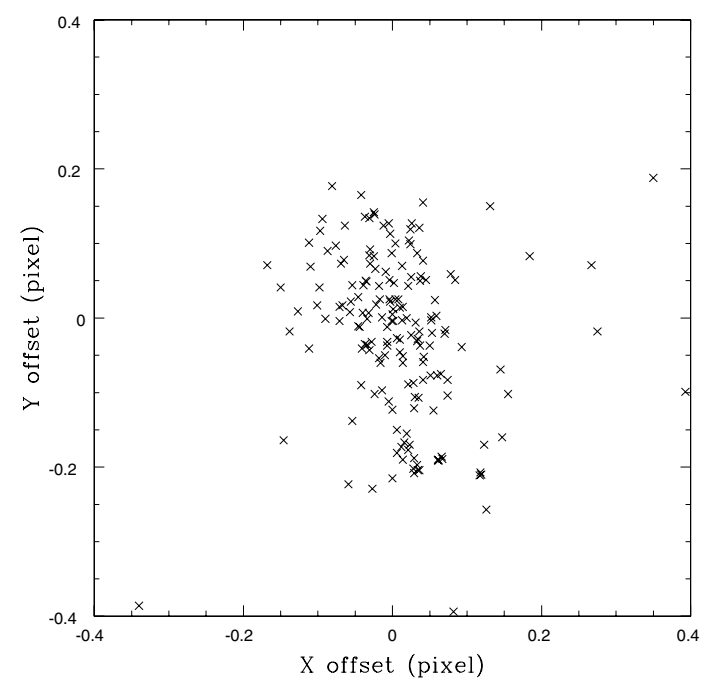

Fig. 3. Position offset for 178 point/point-like sources between two aligned images. The offsets are in the coordinates of WF3 chip. The median value in $X$ is 0.006 pixel and the median value in $Y$ is 0.004 pixel.

Figure 3 shows the distribution of the position offsets of 178 point/point-like sources in two aligned images for 11 CFRS fields and 28 GSS fields. The median of the $X$ offset is 0.006 pixel with a corresponding semi-inter-quartile range (SIQR) of 0.036 pixel and the median of the $Y$ offset is 0.004 pixel with an SIQR of 0.070 pixel. The figure shows that the systematic offsets have been corrected. The center offset, $\sqrt{\Delta x^{2}+\Delta y^{2}}$, can be used to measure the uncertainty of an alignment. For the 178 point/point-like sources, the mean value of the center offset is 0.117 pixel. This denotes that the images are well aligned and can be used for generating color maps.

Many sources do show a center offset in different band images. Figure 4 shows the center offset against the distance from the chip center for all sources (left panel) with $I_{814}$ brighter than $22 \mathrm{mag}$, compared to the distribution for point/pointlike sources (right panel). It is clear that a) the offset is free of the position-dependent effect (e.g. geometric distortion); b) a substantial fraction of sources show a large center offset (up to a few pixels), as shown in the histogram of the center offset (middle panel). In addition, we also plot the center offset distribution for our ISOCAM-detected sample (see Sect. 5.1). On average, ISOCAM galaxies present larger offsets than other sources, which can be related to their intrinsic morphological properties. We use the software Sextractor (Bertin \& Arnouts 1996) to extract source catalogs, including central positions and integrated fluxes. For galaxies with complex morphologies, the central positions given in Sextractor are not always their brightness peaks. An aperture of $3^{\prime \prime}$ is adopted in our photometry. The updated Charge Transfer Efficiency (CTE) correction (Dolphin 2002) and updated photometric zeropoints are adopted in the WFPC2 photometry calibration in the Vega system (Dolphin 2000).

\section{Color map}

To obtain the color map image of an extended source, a key point is to quantitatively select the pixels in the color map with reliable color determination. Instead of adopting a semiempirical noise model (Williams et al. 1996), a method is developed to obtain the signal-to-noise $(S / N)$ ratio of the color map image pixel-by-pixel. Using this $S / N$ ratio image, the color map area can be constrained for the extended source. Adopting an approximation that the Poisson noise distribution function in an HST image is close to a log-normal law, we can obtain that for two images with signals $\mu_{\mathrm{F}_{\mathrm{B}}}, \mu_{\mathrm{F}_{\mathrm{R}}}$ and noises $\sigma_{\mathrm{F}_{\mathrm{B}}}^{2}$, $\sigma_{\mathrm{F}_{\mathrm{R}}}^{2}$, the noise of the their color image satisfies

$\sigma^{2}=\log \left(\frac{\sigma_{\mathrm{F}_{\mathrm{B}}}^{2}}{\mu_{\mathrm{F}_{\mathrm{B}}}^{2}}+1\right)+\log \left(\frac{\sigma_{\mathrm{F}_{\mathrm{R}}}^{2}}{\mu_{\mathrm{F}_{\mathrm{R}}}^{2}}+1\right)$.

Here, the signals and the noises include the ones from both source and sky background. A detailed explanation can be found in the Appendix. The inverse of the noise is proportional to the $S / N$ ratio. Applying this formula to blue and red images pixel-by-pixel, an $S / N$ ratio image associated with the color image can be obtained. This $S / N$ ratio image is indeed for the "color image" of source+sky background. In the pixels in which the source signal is weak, the noise is dominated by that from the sky background. In the $S / N$ ratio image, the fluctuation of the background reflects the uncertainty in the color map image caused by the sky background noise. The color map pixels with an $S / N$ ratio value much higher than the mean $S / N$ ratio background should be much less affected by the noise from the sky background and have a reliable color determination. A criterion of $4 \sigma$ above the mean background of the $S / N$ ratio image is adopted as the detection threshold in the color map image. The color map is given in the HST Vega system and zero background is set in the area below the threshold.

The $S / N$ ratio image can give a quantitatively measurement of the reliability of each pixel in the color map image. The $S / N$ ratio image does not introduce any bias against very red or very blue color regions because it accounts for both the $S / N$ of the two WFPC2 images. The $S / N$ ratio image is shown with $V_{606}$ and $I_{814}$ images in three typical cases, elliptical galaxy (Fig. 5), spiral galaxy (Fig. 6) and merging system 

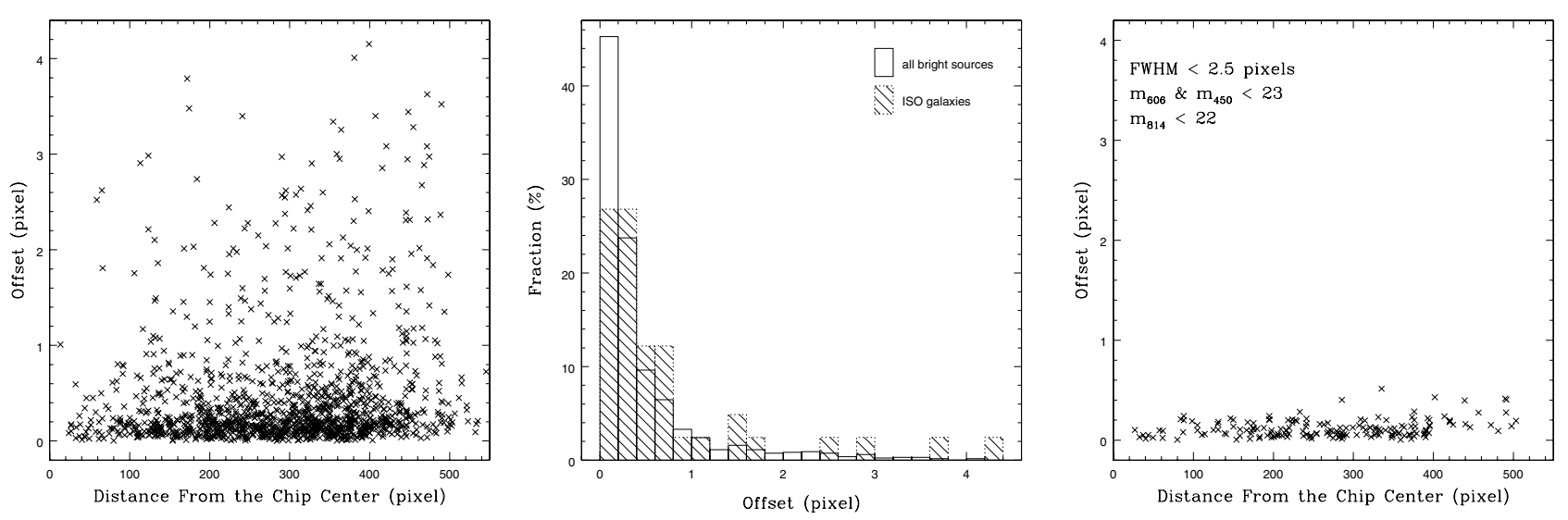

Fig. 4. Left panel: center offset of the bright sources $\left(m_{814} \leq 22\right)$ between blue $\left(V_{606}\right.$ or $\left.B_{450}\right)$ and red $\left(I_{814}\right)$ image as a function of their positions in WFPC2 WF chip, expressed as the distance from the chip center. Middle panel: histograms of the center offset for all bright sources and for our ISOCAM-detected galaxies. Right panel: center offset for the point/point-like sources. In this panel, the mean value of the offset is 0.117 pixel with $\sigma=0.088$ pixel.
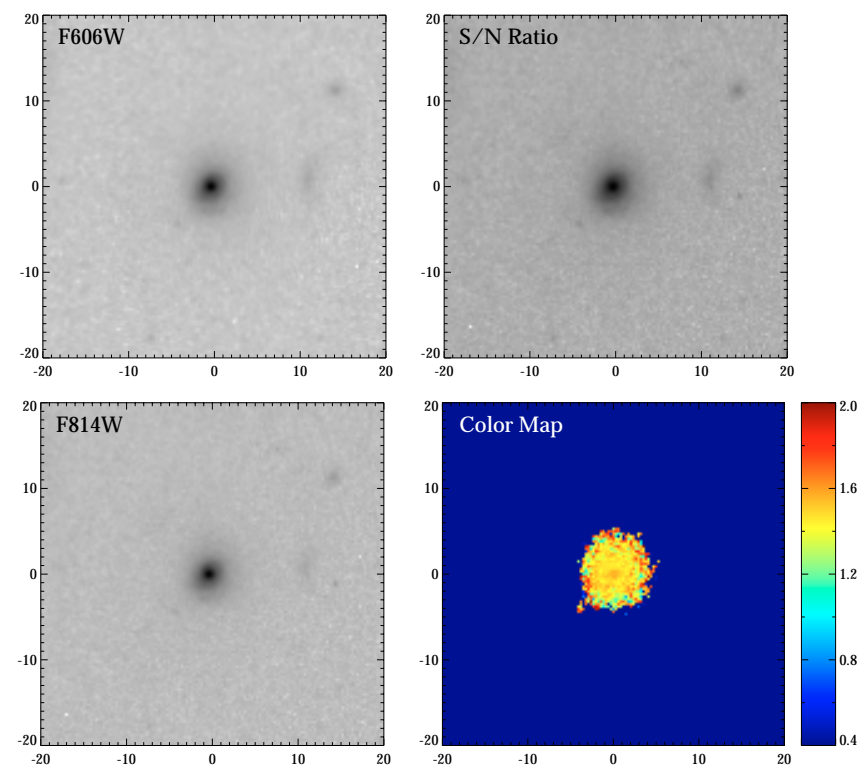

Fig. 5. $V_{606}, I_{814}$, the color map $S / N$ ratio, as well as color map images of elliptical galaxy 03.0037 at redshift 0.1730 . (See the electronic edition for a color version of this figure.)

(Fig. 7). The resulting color map image with the $S / N$ ratio image is displayed. The color bar next to the color map shows the color scheme in the observed frame. The color range is adjusted for best visualization. Each image is labeled top-left corner. In Figs. 5 and 6, each image has a size of $40 \times 40 \mathrm{kpc}$ while in Fig. 7 the size is $60 \times 60 \mathrm{kpc}$.

\section{Morphologies of LIRGs}

\subsection{LIRG sample}

The 17 HST imaging fields cover about 87 of the 200 square arcminute area of the CFRS fields $0300+00$ and $1415+52$. We obtained either $B_{450}-I_{814}$ or $V_{606}-I_{814}$ color maps for 265 galaxies $\left(I_{\mathrm{AB}}<22.5\right)$. Among them, 169 have spectroscopic redshifts given in the CFRS redshift catalog or in
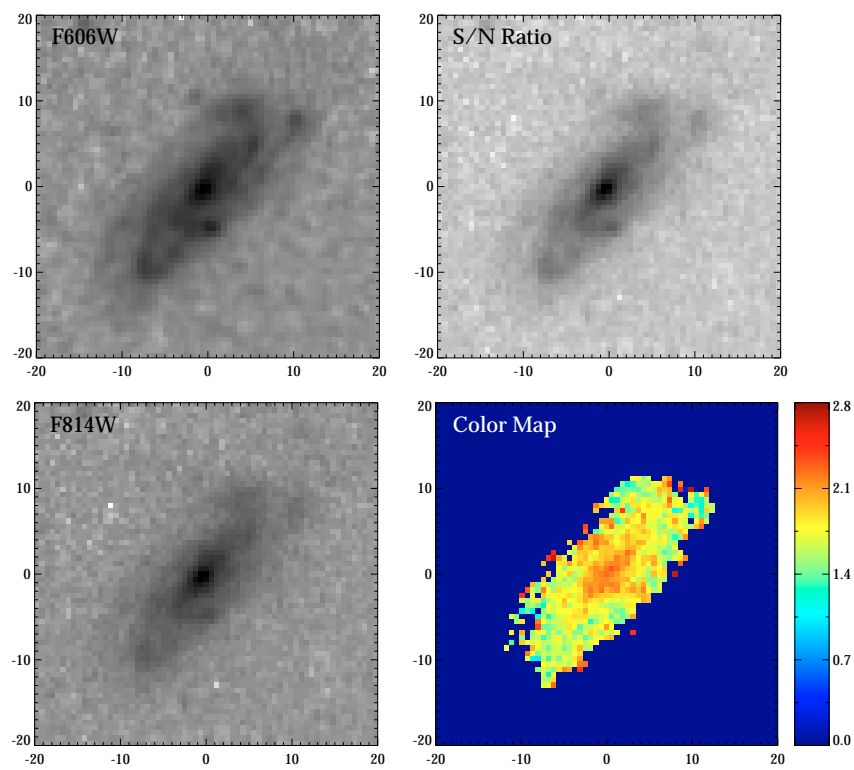

Fig. 6. Same as in Fig. 5 for disk galaxy 03.0046 at redshift 0.5120 . (See the electronic edition for a color version of this figure.)

the literature. ISOCAM observations detected 60 objects and 77 objects brighter than $300 \mu \mathrm{Jy}$ at $15 \mu \mathrm{m}$ with $S / N>5$ in the CFRS fields $0300+00$ and $1415+52$, respectively. Of the 137 objects, a color map is available for 33 objects in the $0300+00$ field and 26 objects in the $1415+52$ field. Of the 137 ISOCAM-detected objects, $82.5 \%$ (113) have optical counterparts brighter than $22.5 \mathrm{mag}$ and $94.2 \%$ (129) brighter than $23.5 \mathrm{mag}$ in the $I_{\mathrm{AB}}$ band. For objects fainter than $I_{\mathrm{AB}}=22.5 \mathrm{mag}$, the imaging sample points are limited for deriving reliable morphological parameters and color distribution. Therefore they are not included in this analysis. Since the vast majority of ISOCAM-detected objects are indeed optically bright, this would not cause a significant bias. Note that the fraction of ISOCAM sources imaged by HST (59 among $113 I_{\mathrm{AB}}<22.5$ galaxies, 52.2\%) is larger than the area fraction of HST imaging of the two 

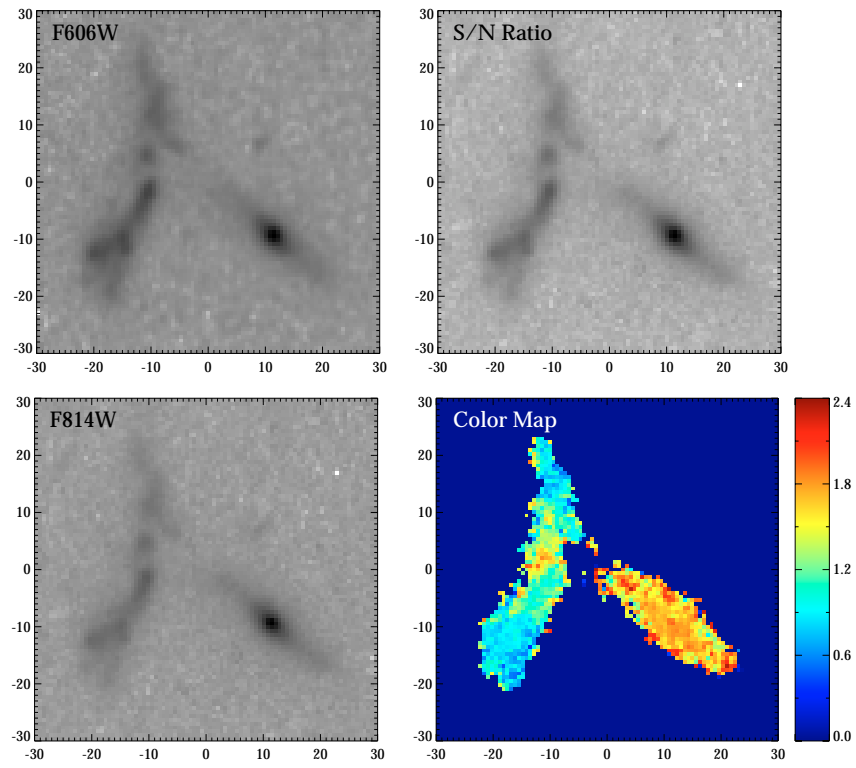

Fig. 7. Same as in Fig. 5 for merging system 03.1309 at redshift 0.6170. (See the electronic edition for a color version of this figure.)

CFRS fields (87 vs. 200 square arcminute, 43.5\%), because our survey includes complementary data aimed at investigating ISOCAM source morphologies.

In the 59 ISOCAM-detected galaxies, 53 objects have known redshifts, including 2 objects at $z>1.2$ which have been removed from our sample.

Table 3 lists the complete sample of ISOCAM galaxies imaged by HST in two bands. The objects are organized into three groups, 6 with unknown redshift, 15 in the nearby $(z \leq 0.4)$ and 36 in the distant $(0.4<z<1.2)$ universe. Nearby galaxies are not IR luminous $\left(L_{\mathrm{IR}} \sim 10^{10} L_{\odot}\right)$ and can not be taken as the local counterparts of the distant LIRGs. The 36 distant objects are used to reveal the morphological properties of the distant LIRGs. In each group, the objects are tabulated in the order of their CFRS identifications. In Table 3, the apparent magnitudes $m_{450}$ (Col. 3), $m_{606}$ (Col. 4) and $m_{814}$ (Col. 5) are given in HST Vega system. An aperture of $3^{\prime \prime}$ is adopted for the HST image photometry. The absolute $B$ band magnitude (Col. 6) and $K$ band magnitude (Col. 7) are provided in the $\mathrm{AB}$ system, using isophotal magnitudes from the groundbased imaging (Lilly et al. 1995). The K-correction is calculated based on ground-based $B, V, I$ and $K$ band CFRS photometry (see Hammer et al. 2001 for details). The IR luminosity is given in Col. 8. Three models are used to calculate the IR luminosity and derive proper uncertainties (see Flores et al. 2004 for details). Figure 8 shows the IR luminosity distribution for the 36 distant LIRGs.

\subsection{Morphological classification}

Based on HST imaging, two-dimensional fitting has been carried out to derive the structural parameters used to quantify the morphological features. With the structural parameters and information derived from color maps, the morphological classification was performed by two researchers independently.

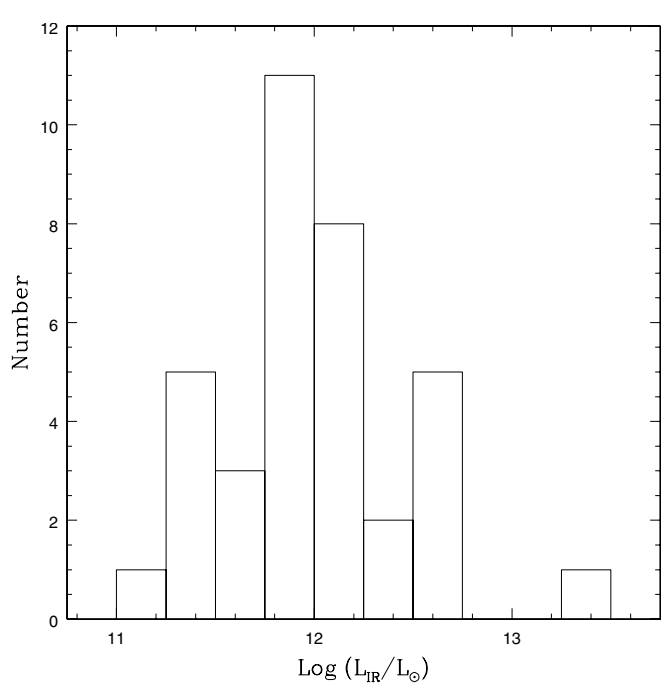

Fig. 8. Histogram of the IR luminosity for 36 distant LIRGs.

\subsubsection{Structural parameters}

The two-dimensional fitting is performed using the software GIM2D (see Simard et al. 2002 for more details). Two components, bulge and disk, are used to fit the surface brightness distribution. The fractions of bulge luminosity in total $B / T$ and $\chi^{2}$ are listed in Table 3 (from Cols. 9 to 14). The parameter $B / T$ is correlated with the Hubble type, increasing for earlytype galaxies. In addition to the "quality" parameter $\chi^{2}$, the residual image, which is the difference between the observed image and the modeled image, is also used to estimate the quality of the fit. Good fitting is characterized by a $\chi^{2}$ close to unity and a residual image with little random-distributed residual emission. However, in the case of a spiral galaxy with visible arms, the residual image exhibiting regular arms will refer to the fitting as good even though the $\chi^{2}$ value is biased to be different from unity due to the presence of arms. The inclination angle of the disk is also derived from the two-dimensional fitting.

\subsubsection{Color distribution properties}

With information provided by color distributions, morphological classification can be improved dramatically. The appearance in a color map is free from the arbitrary adjustment in visualization of an image. Physical properties can be derived from the color map including whether some regions are dusty or star-forming. Apart from the usual morphological classification based on the brightness distribution, the color information provides a new way to compare distant galaxies with local galaxies in the Hubble sequence.

We obtain color map for each LIRG in the observed frame. Instead of applying K-correction and deriving the color in the rest frame, we compare the observed color with the modeling color. Figure 9 illustrates the modeled color-redshift curves for $V_{606}-I_{814}$ and $B_{450}-I_{814}$. Using GISSEL98 (Bruzual \& Charlot 1993), the observed colors in the HST Vega system are given at different redshifts for four galaxy models, corresponding to elliptical (single burst), S0 ( $\tau=1 \mathrm{Gyr})$, Sbc $(\tau=7 \mathrm{Gyr})$ and 

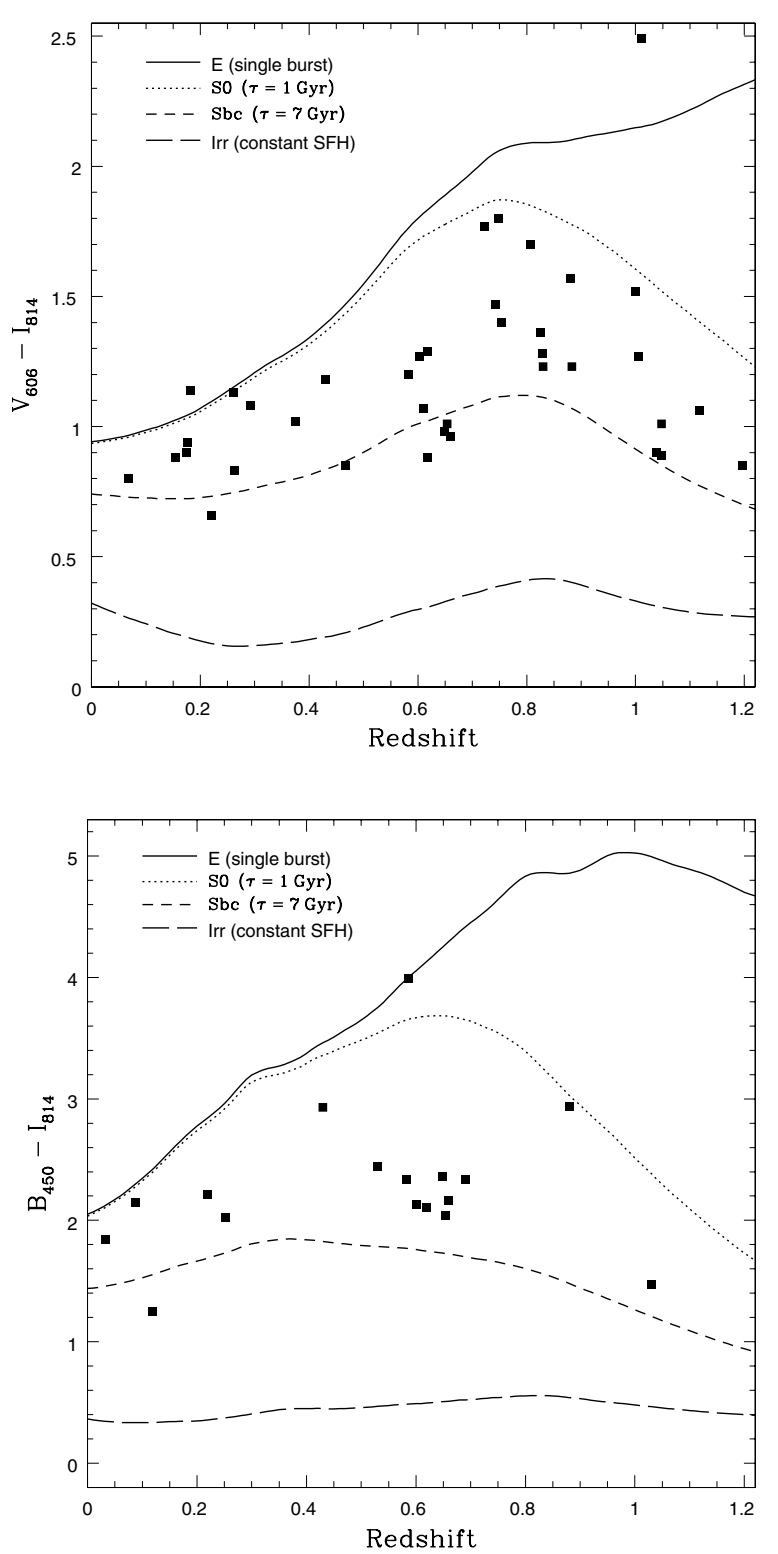

Fig. 9. $V_{606}-I_{814}$ (top) and $B_{450}-I_{814}$ (bottom) observed colors for LIRGs as a function of redshift. Similar to Fig. 6 in Menanteau et al. (2001), four solar metallicity, formed at $z=5$ galaxy models, including an Elliptical galaxy (single burst), S0 galaxy ( $\tau=1 \mathrm{Gyr}$ ), Sbc galaxy $(\tau=7 \mathrm{Gyr})$, irregular galaxy (constant SFR) are present for a comparison. Almost all LIRGs are redder than Sbc galaxy.

irregular galaxies (constant star formation rate with a fixed age of $0.06 \mathrm{Gyr}$ ). We assume a formation epoch at a redshift of $z=5$. Distant LIRGs are compared with the models using their integrated colors. Almost all LIRGs are redder than an Sbc galaxy. Using the modeled color-redshift relations, we investigate the colors of specified regions. We refer to the region in the outskirts as red as or redder than an elliptical galaxy as a dusty region and the region off the central area bluer than an Sbc galaxy as a star-forming region.

\subsubsection{Morphology labels}

By visually examining galaxy morphologies, we have tried to label each target with its "Hubble type". For the galaxies well fitted by a bulge+disk two-dimensional structure, we divide them into five types in terms of the fraction of bulge luminosity in total: E/S0 $(0.8<B / T \leq 1)$, S0 $(0.5<B / T \leq 0.8)$, $\mathrm{Sab}(0.15<B / T \leq 0.5)$, $\mathrm{Sbc}(0<B / T \leq 0.15)$ and $\mathrm{Sd}(B / T=$ $0)$. We also introduce three additional types to describe the compact (C) galaxy which is too concentrated to be decomposed, irregular (Irr) galaxy and "tadpole" (T) galaxy. A quality factor is provided to represent our confidence of our classification: 1 - secure, 2 - possibly secure, 3 - insecure and 4 - undetermined. We also provide a classification for galaxies that show signs of interacting or merging: M 1 - obvious merging, M 2 - possible merging, I1 - obvious interaction, I2 - possible interaction and $\mathrm{R}$ - relics of merger/interaction.

The experienced eye is essential to classify the objects differing significantly from the adopted models. With information from the two-dimensional structure fitting and the color map, the visual examination is carried out by two of the authors F.H. and X.Z.Z. independently, to reduce arbitrariness. The classifications are consistent with each other for most objects. After fully discussing a few objects, a final morphological classification for the LIRG sample is made. In Table 3, galaxy type (Col. 15), quality factor (Col. 16) and Interaction/merging type (Col. 17) are listed.

\subsection{Individual descriptions}

In Fig. 10, color maps (right) of the 36 LIRGs are shown, along with $I_{814}$ negative greylevel images (left). The images are given in order of CFRS identification (from left to right, top to bottom). The color bar in each color map shows the color range. The same color ranges over -1 to 3 and 0 to 4 are applied to all objects in $V_{606}-I_{814}$ and $B_{450}-I_{814}$, respectively. For the 36 LIRGs, the morphologies as well as the color distributions are remarkably different. A description of each target is present in turn.

03.0035 This galaxy has a bright bulge surrounded by diffuse emission. Spiral arm-like structures or tidal tails are perceptible in the diffuse region. The color map shows that the bulge has a color comparable to that of an S0 galaxy. Dusty regions and star-forming regions are revealed in the color map.

03.0062 Its clear and regular spiral arms surrounding a spheroid core as well as its round appearance suggest that this is a face-on spiral galaxy. The color map confirms the red central region to be a bulge with color close to that of an S0 galaxy. Star-forming regions are distributed along the arms.

03.0085 This is an edge-on spiral galaxy $\left(i \sim 75^{\circ}\right)$. This galaxy was imaged close to the chip border and the light distribution was not completely recorded. However, the central bright region is not a bulge. The color map exhibits symmetric arc structures with color close to that of an irregular galaxy, suggesting star formation in the disk. 

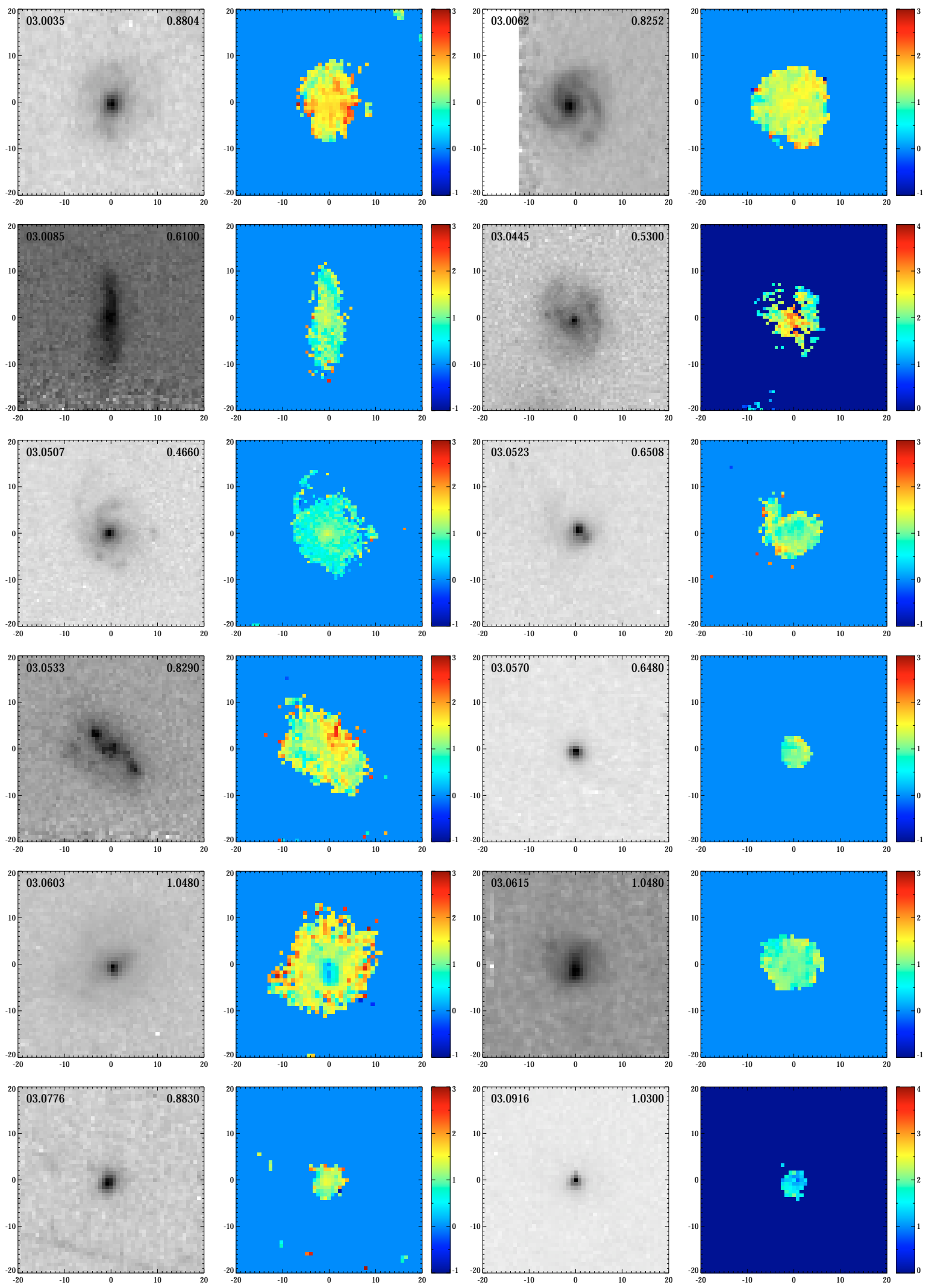

Fig. 10. $I_{814}$ band and color map images of distant LIRGs. For each target, the name and redshift are labeled top-left and top-right in the $I_{814}$ image. The color bar ranges from -1 to 3 for the $V_{606}-I_{814}$ color map and 0 to 4 for the $B_{450}-I_{814}$ color map. The blank in $I_{814}$ image is due to the target imaged close to the chip border. The size of each image is $40 \times 40 \mathrm{kpc}$ except for the object 03.1309 , which is $60 \times 60 \mathrm{kpc}$ to display the whole merging system. (See the electronic edition for a color version of this figure.) 

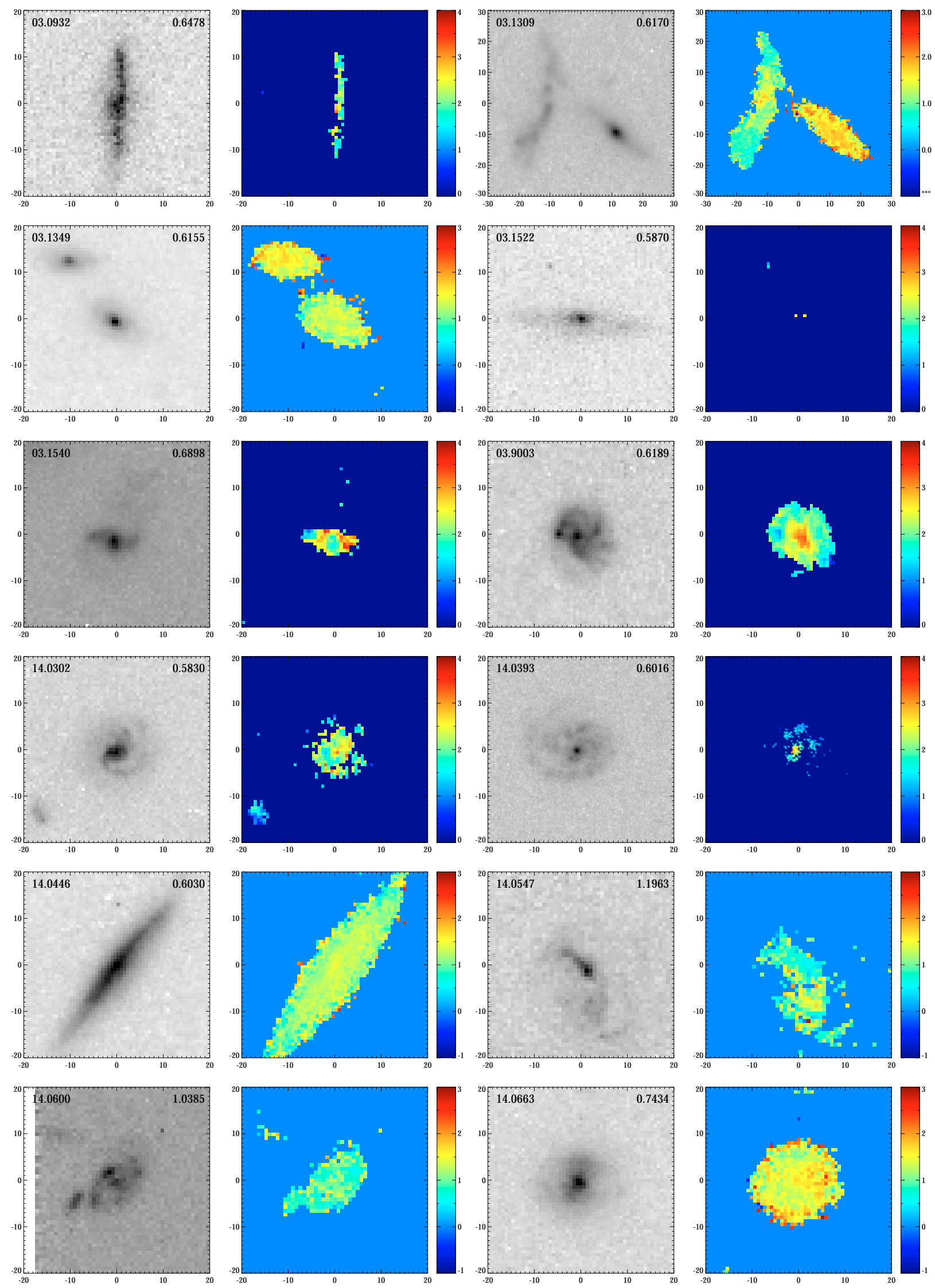

Fig. 10. continued. 

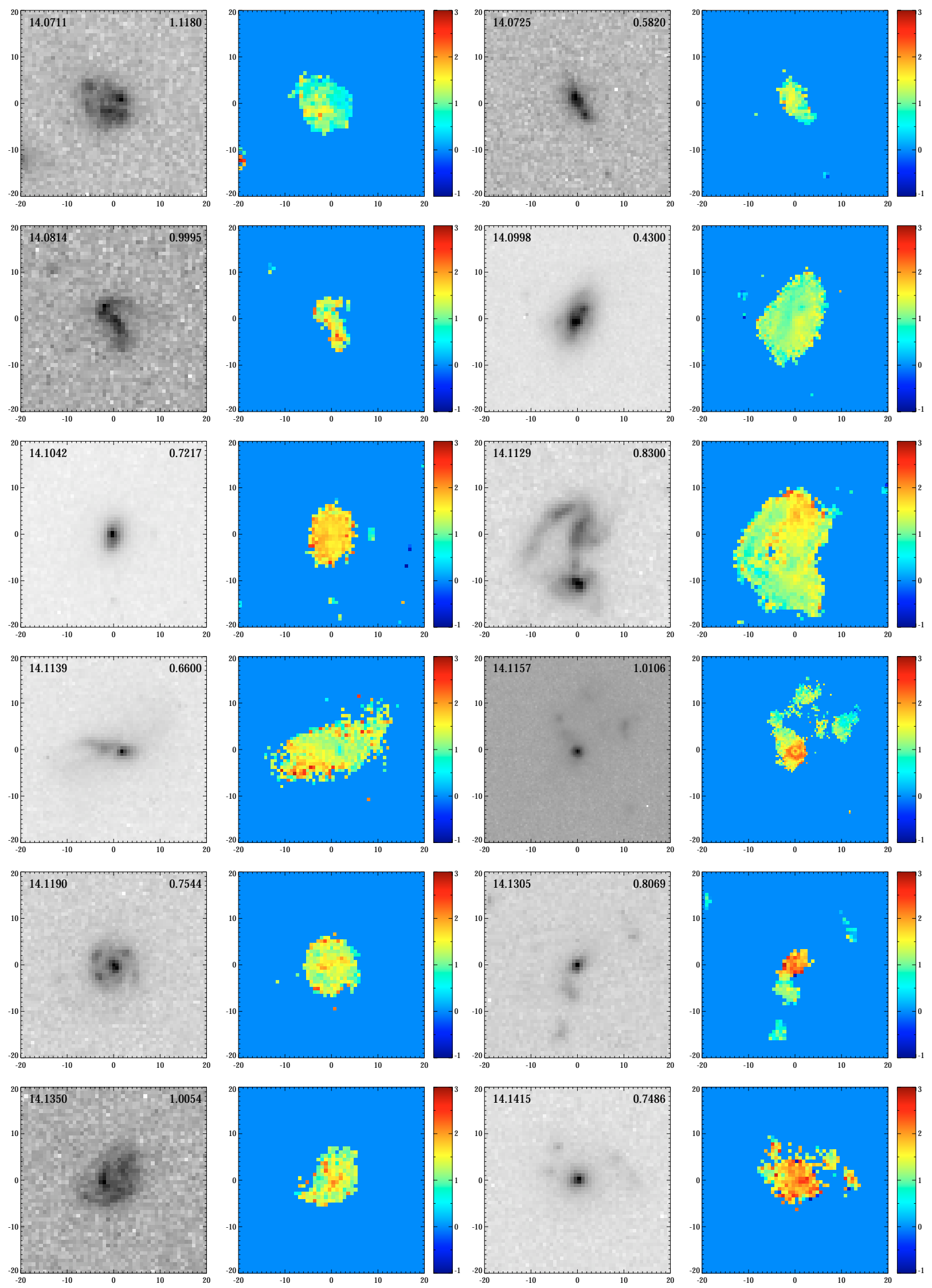

Fig. 10. continued. 
03.0445 WFPC 2 image and color map show a spheroidal component in the center surrounded by blue diffuse emission. Arm structures are visible in the surrounding regions. This galaxy is classified as an Sbc galaxy. The arms are asymmetric and it is not clear whether such asymmetric arms are related to an interaction/merger event.

03.0507 This is a face-on spiral galaxy with a red bulge surrounded by blue diffuse emission. Arm structures and starforming knots can be seen around the red bulge. A striking long filament, different from a spiral arm, could be associated with a stripe remnant of an infalling dwarf galaxy.

03.0523 This is a "tadpole" galaxy. It is widely believed that such a "tadpole" feature is an indicator of a major merger in an advanced phase. The tidal tail is clearly seen in the WFPC2 image. The color map exhibits a conspicuous color gradient that the central region is bluer than the outer region. The concentrated light distribution suggests this object is a compact galaxy.

03.0533 The morphology of this galaxy is complex and shows multiple components. A clumpy structure suggests an irregular classification. It is however possible that this galaxy is a nearly edge-on spiral galaxy, with a long tidal tail roughly parallel to the disk. If this is the case, the tidal tail is bluer than the disk. The central region of this spiral galaxy is dusty and some star-forming regions are detected along the arms and the long tidal tail.

03.0570 This is a compact galaxy with an integrated color close to that of an Sbc galaxy. The light distribution is dominated by a bright and compact nuclear component.

03.0603 An extremely blue core dominates this galaxy and red diffuse emission extends by about $10 \mathrm{kpc}$. The blue core is even bluer than an irregular galaxy. A broad Mg II $\lambda 2799$ line is detected with CFHT spectroscopy. This galaxy is classified as a compact galaxy. However, the faint surrounding region appears round. This object could be a galaxy with a very faint face-on disk.

03.0615 This galaxy is compact and with a round and smooth morphology. The global color is comparable to that of a spiral galaxy earlier than Sbc type. A star-forming region is visible in the color map $5 \mathrm{kpc}$ from the nucleus.

03.0776 The morphology of this galaxy is elongated and compact. Distorted fuzzy emission is detected. The color map reveals a relatively red core surrounded by blue regions. The integrated color of this galaxy is close to that of an Sbc galaxy.

03.0916 This is a compact galaxy. The light distribution is too compact to obtain the structural parameters. Broad emission lines detected in CFHT spectroscopy suggest that this galaxy is a type I AGN.

03.0932 From the WFPC2 $I_{814}$ image, we can see that this is an extremely edge-on galaxy with a bulge in the center. However, the bulge is not detected in the $B_{450}$ band image.

03.1309 This chain galaxy is a merging system. In this system, the edge-on disk galaxy has a dominant bulge with a color close to that of an elliptical galaxy. Along the chain, starformation regions are visible.

03.1349 The regular appearance and a dominant bulge suggest an Sab classification for this galaxy. The bulge is compact and relatively blue, comparable to the disk. Our spectroscopy confirms that the companion galaxy $20 \mathrm{kpc}$ away is an interacting system. .

03.1522 This is an edge-on spiral galaxy. It is not detected in the deep WFPC2 $B_{450}$ band (6600 s exposure time) implying a heavy extinction (dust screen). CFRS ground-based photometry reveals that this object is 0.3 mag redder than an elliptical galaxy at the same redshift.

03.1540 This is a compact galaxy. The luminous component is surrounded by a fuzzy extended region (a disk?). The compact component looks like a giant bar. The color map reveals a distinct blue core surrounded by dusty regions. Off the blue core, a blue region appears at one end of the bar. The strong IR emission should relate to the central starforming regions. While the starburst can be triggered by the instability of a bar, it is also conceivable to ascribe the distinctive morphology to merger/interaction.

03.9003 The brightness distribution of this galaxy is quite complex and irregular. From the color map, it can be seen that blue star-forming regions surround a red central region (a bulge?).

14.0302 This galaxy is a face-on spiral galaxy with clear arms and a bulge/bar detected in the WFPC2 image. In the color map, star-forming regions are revealed surrounding the center and the arms.

14.0393 This is a face-on spiral galaxy with star formation spread over the arms. This galaxy is very extended and has a small bulge which is distinctive in the color map $1.5 \mathrm{mag}$ redder than that of the star-forming regions on the arms.

14.0446 This galaxy is a nearly edge-on $\left(i \sim 80^{\circ}\right)$ pure disk galaxy. The color map confirms that no red central region similar to a bulge is detected. This disk galaxy is rather a giant characterized by a disk scale length of $6.8 \mathrm{kpc}$ in the $I_{814}$ band. The mean $V_{606}-I_{814}$ color is close to that of an Sbc galaxy at that redshift.

14.0547 The heavily distorted morphology showing two main components and tidal tail features suggests that this is a pair system undergoing a major merger episode. One component is elongated and blue, while the other is relatively red.

14.0600 This galaxy is a system with complex morphology and clumpy light distribution. The clumpy knots are bluer than an Sbc galaxy, and are associated with star formation. While it is classified as an irregular galaxy, this galaxy is probably undergoing a minor merger event.

14.0663 This galaxy shows a round appearance. The central region exhibits a peanut-shaped structure, which could be linked to a giant bar. The color distribution shows blue regions surrounding both ends of the "peanut".

14.0711 The complex, clumpy structure seen in the WFPC2 image suggests an irregular galaxy. Dust regions and star-forming regions are revealed in the color map.

14.0725 This galaxy has an elongated morphology and shows a color gradient. The color map shows two distinct regions. The large region has a color close to that of an elliptical galaxy while the small region is as blue as an Sbc galaxy. It is not clear whether the elongated morphology is due to two 
merging galactic nuclei since no tidal tail features appear in the surrounding diffuse emission.

14.0814 This is a system with an S-shape structure. This galaxy could be a barred spiral rather than a major merger because it shows no evidence of two components. While it is labeled as an irregular galaxy, it could be associated with relics of merger/interaction.

14.0998 This system has a peculiar morphology composed of two very close components surrounded by diffuse emission. Peculiar substructures like two tidal tails can still be seen. The minor component is bluer than the major one by about $1 \mathrm{mag}$. The color distribution of this galaxy is quite complex. Broad dusty regions and star-forming regions can be seen in the color map.

14.1042 A round, smooth and elongated morphology can be seen from the WFPC2 image. This galaxy is as red as an elliptical galaxy. but its brightness distribution is more concentrated.

14.1129 This system consists of two comparable components separated by $12 \mathrm{kpc}$. Strikingly, a distinct tidal tail and a bridge connecting the two components suggest that this system is undergoing a major merger episode. The tidal tail is bluer than the two galactic nuclei.

14.1139 From the WFPC 2 image, a peculiar appearance showing the merging of two galaxies can be seen. The two galactic nuclei are as close as $3.5 \mathrm{kpc}$, surrounded by diffuse and extended emission. Substructures like tidal tails are visible in the surrounding regions. The major component has a blue core.

14.1157 This is a system composed of multiple components. Material streams connecting the components are detected. The brightest component is very red. This object is detected by the PC chip in the GSS very deep field. The higher resolution color map reveals a ring structure associated with a point source in a very red region, suggesting a heavily obscured AGN inside it.

14.1190 The morphology of this galaxy is round and clumpy. Spiral arms are visible around the center with several bright blue knots. There is no dominant central component and this galaxy is classified as an Sd galaxy.

14.1305 This system is a merging system showing a dominant component and a conjoint one. Seen in the color map, the dominant component is even redder than an elliptical galaxy, suggesting that it is very dusty. Within $20 \mathrm{kpc}$, there are two other components with colors comparable to that of an Sbc galaxy. It is unclear whether they belong to the same physical system.

14.1350 This galaxy is classified as an irregular galaxy due to its complex morphology and irregular light distribution. The bright components/knots are as blue as an Sbc galaxy and the central region is very red (dust?).

14.1415 Dominated by a major component, this galaxy is classified as a compact galaxy. The central dominant component is surrounded by diffuse emission. Four minor components are seen in the surrounding emission, which suggests that this galaxy is possibly the relic of a merger event. The main component is dusty.

\section{Discussion}

\subsection{Morphological properties}

From Table 3, we can derive the global morphological properties for distant LIRGs. Of the 36 galaxies, 13 (36\%) are classified as disk galaxies with "Hubble type" from Sab to Sd. For these disk galaxies, their morphological classifications are secure $(Q<2)$. The object 03.1522 is an extremely red edgeon disk galaxy. This galaxy is so red that almost no light is detected in the $B_{450}$ band, which leads to few pixels available in its color map. We label this galaxy simply as "Spiral" due to the large uncertainty in determining the bulge fraction. Of the 13 disk galaxies, 4 of them are very edge-on. Morphological classification shows that $25 \%$ (9 in 36) of the LIRGs are compact galaxies as they show concentrated light distribution (see the definition in Hammer et al. 2001). Such a high fraction is strikingly similar to that derived from optical samples (Guzmán et al. 1997; Hammer et al. 2001). Their two-dimensional structure fitting suffers from large uncertainties. Of the 36 galaxies, $8(22 \%)$ are classified as irregular galaxies which show complex morphology and clumpy light distribution. In the 36 LIRGs, only 6 cases $(17 \%)$ are major ongoing mergers showing multiple components and apparent tidal tails. Five (either compact or irregular) LIRGs are possibly linked to merging (labeled by M 2) and one spiral LIRG is probably an interacting system (labeled I2). Signs of merging or relics of interactions (possibly) occur in 9 LIRGs (labeled R and R?). Accounting for them and for major mergers, the total fraction of merging/interacting systems is estimated to be $58 \%$ (21 of 36) of the LIRGs.

For galaxies at redshift $\sim 1$, the cosmological dimming effect becomes significant and the morphological classifications might have some uncertainties since faint features are barely detected at the detection limits of our WFPC2 imaging data. Some irregular galaxies could be spiral galaxies. The objects classified as spiral galaxies and major mergers are free from those uncertainties because their main structural properties have already been determined. Hence, in the present LIRG sample, the fraction of spiral galaxies is well estimated or slightly underestimated if there are spirals misclassified as irregular galaxies. HST $I_{814}$ band imaging is available for all LIRGs. Thus the compactness of each galaxy can be well determined and the fraction of the compact galaxies is reliable (see e.g. Hammer et al. 2001).

\subsection{LIRGs: Massive systems related to large disks?}

$K$ band luminosity is widely used to estimate the stellar mass. For our 36 sample LIRGs, $K$ band luminosity is available for 24 of them. Following Hammer et al. (2001), we assume a unity mass-to-luminosity ratio in the $K$ band and estimate the stellar masses for the 24 LIRGs. The derived stellar masses range from $1.4 \times 10^{10}$ to $2.9 \times 10^{11} M_{\odot}$, compared to $1.8 \times 10^{11} M_{\odot}$ for the Milk Way mass. Note that extinction and age of stellar populations are not considered in estimating the stellar mass 


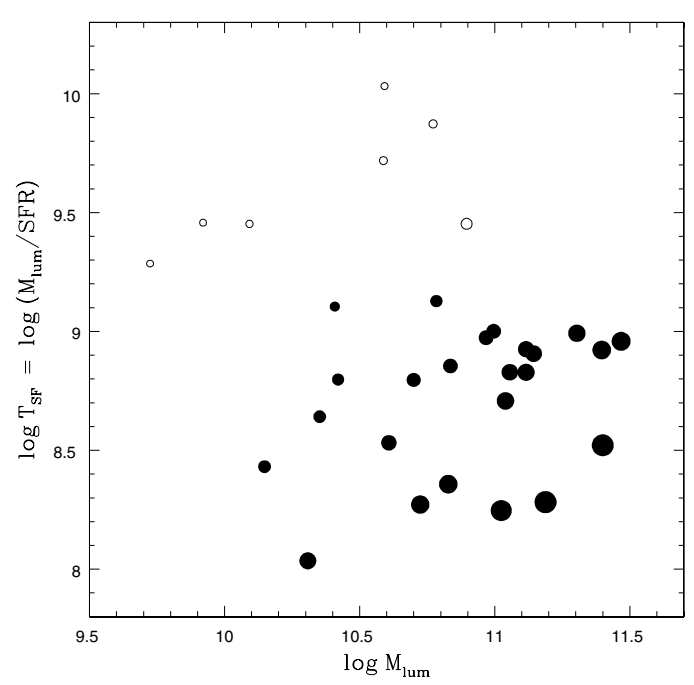

Fig. 11. Stellar mass versus time scale to duplicate the bulk of stars at a rate of the observed value. The stellar mass is derived from $K$ band luminosity. The size of the point is scaled by the SFR. Nearby galaxies listed in Table 3 with available $K$ band luminosity are shown with open circles for comparison.

due to the numerous related uncertainties (e.g. those associated with the assumed stellar population mixing and the dust extinction modeling). We notice that the stellar masses we obtained are, systematically, from $50 \%$ to $100 \%$ of the values derived by Franceschini et al. (2003), after a careful examination of galaxies with similar $K$ band luminosities. Indeed, Franceschini et al. (2003) have derived stellar masses using spectral synthesis modeling of the overall optical-IR continuum. Further discussion about uncertainties in the mass estimate can be found in Berta et al. (2004). Adopting $M_{\mathrm{AB}}^{*}(K)=$ -21.82 for $H_{0}=70$ (Glazebrook et al. 1995), LIRGs are systems ranging from $0.3 L^{*}$ to $6.7 L^{*}$ with median value $2.2 L^{*}$. These LIRGs were undergoing violent star formation at rates of the order of $\sim 100 M_{\odot} \mathrm{yr}^{-1}$. They may add a significant mass contribution over a short time. For a galaxy, the ratio of stellar mass to SFR is the time scale for the formation of the bulk of the stars. If star formation is sustained at the observed rate, LIRGs could duplicate themselves within $10^{8}$ to $10^{9} \mathrm{yr}$ (Fig. 11).

A large fraction of LIRGs are classified as disk galaxies. In Table 3 , the disk scale length $R_{\mathrm{d}}$ is also listed for the LIRGs classified as spiral galaxies. We can see that all the LIRG disks are large galaxies with $R_{\mathrm{d}} \geq 2.9 \mathrm{kpc}$ (corresponding to $4 \mathrm{kpc}$ at $H_{0}=50$ ) except for the object 14.1190 , which is a face-on Sd galaxy from the GSS fields. We suspect that its disk scale length has been severely underestimated due to the shallow detection $\left(\sim 24.5 \mathrm{mag} \operatorname{arcsec}^{-2}\right.$ in the $I_{814}$ band, compared to $\sim 25.5 \mathrm{mag} \operatorname{arcsec}^{-2}$ in other fields). We compare the LIRG disks with the size-selected disk sample using the distribution of the disk central surface brightness. Lilly et al. (1998, L98 hereafter) presented a detailed study of a large disk $\left(R_{\mathrm{d}} \geq 4 h_{50}^{-1} \mathrm{kpc}\right)$ sample, which is homogeneous and essentially complete. Similar to L98 (see their Fig. 12), we plot the LIRG disks in the diagram of central surface brightness versus redshift. The observed $I_{814}$ band

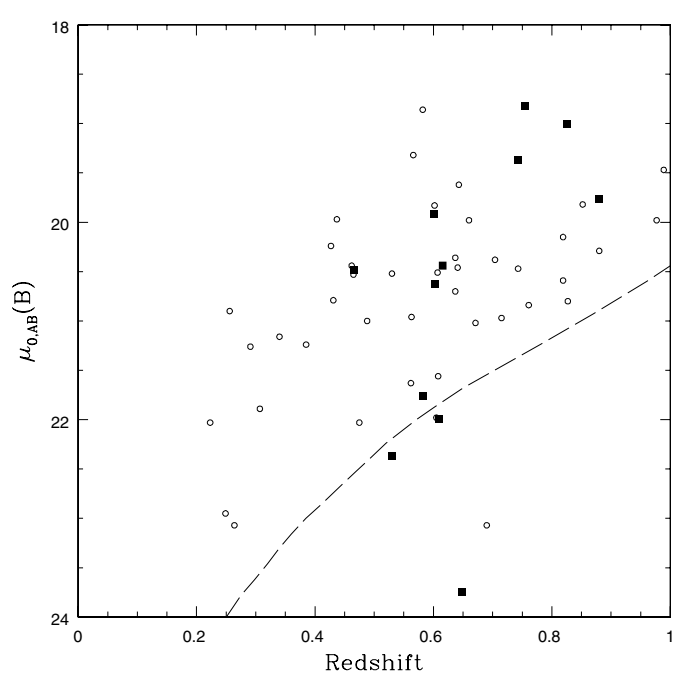

Fig. 12. Central surface brightness of the disk components as a function of redshift for LIRG disks in our sample (solid square), compared with the large disk galaxies in L98 (open circle). The long-dashed line is the selection criterion $\left(I_{\mathrm{AB}}<22.5\right)$ for a pure disk galaxy with scale length $4 h_{50}^{-1} \mathrm{kpc}$.

central surface brightness of the disk components derived from the two-dimensional structure fit is used to obtain the rest frame $B_{\mathrm{AB}}$ band central surface brightness. Cosmological dimming and K-correction are corrected following L98. We use the central surface brightness color $V_{606}-I_{814}$ (or $B_{450}-I_{814}$ ) to calculate the K-correction except for LIRG disks 03.0085, 03.0445, 03.0932, and 14.0393, whose integrated HST colors are adopted because the central surface brightness is either available only in one band or not well estimated in the $B_{450}$ band. The edge-on LIRG disk 03.1522 is not included because of the heavy extinction. A spectral energy distribution is chosen to match the observed color from theoretical ones at different ages for a solar abundance galaxy with e-folding time $\tau=1 \mathrm{Gyr}$ (see Hammer et al. 2001 for more details). As shown in Fig. 12, the central surface brightness of 8 LIRG disks is consistent with that of the large disk galaxies in L98 at similar redshift (LIRG disks 03.0085, 03.0445, 03.0932, 03.1522 and 14.0393 are absent because of no secure structural parameters available in one band). This confirms that LIRG disks belong to the large disk galaxy population.

We also investigated if the size-selected sample of distant disk galaxies includes LIRGs. In L98, 5, 14 and 5 objects were selected from the CFRS $0300+00$ and $1415+52$ fields in redshift bins $0.2<z<0.5,0.5<z<0.75$ and $0.75<z<1.0$, respectively. After a cross-identification with ISOCAM observations, 6 of 19 disk galaxies in $0.5<z<1.0$ are found luminous in the IR band with IR luminosities ranging from $1.6 \times$ to $12 \times 10^{11} L_{\odot}$ (the median value is $5.3 \times 10^{11} L_{\odot}$ ). No galaxy in the redshift bin $0.2<z<0.5$ is identified as a nearby IR luminous or starburst galaxy. Of the 6 IR luminous large disk galaxies, 5 are included in our HST sample, including 3 morphologically classified as spirals, one as a bar-dominated compact galaxy (03.1540), although it shows evidence of an extended faint disk, and one as a merger (14.1139). This indicates that in the large disk galaxy population at redshifts ranging from 0.5 
to 1.0 , about $30 \pm 12 \%$ are infrared luminous. This confirms that LIRG disks are large (massive?) disks.

LIRGs are systems with an average stellar content of $1.4 \mathrm{M}^{*}$, and they are intimately linked to large disks size-selected disks include a significant fraction of LIRGs. We believe that the star formation in large disks (derived from UV measurements) in L98 is severely underestimated. Indeed from their IR luminosities, LIRG disks were forming stars at a high rate, ranging from 18 to $210 M_{\odot} \mathrm{yr}^{-1}$ averaged to $110 M_{\odot} \mathrm{yr}^{-1}$. This is contradictory to the modest star formation rate of about 3-10 $M_{\odot} \mathrm{yr}^{-1}$ reported by L98. This indicates that UV and [OII] luminosities are poor tracers of the star formation rate.

The number density of LIRGs is much larger at $z \sim 1$ than at the present day by a factor of more than 40 (Elbaz et al. 2002). From the above discussions, we believe that they significantly contribute to the large and massive disks population at $z=0.5-1$. Our result is contradictory to that of Brinchmann \& Ellis (2000), who claimed that dwarf galaxies, rather than massive systems, were responsible for the star formation activity since $z \sim 1$. As an example, we assume that the L98 SFR of $\sim 7 M_{\odot} \mathrm{yr}^{-1}$ apply to the large disks which have not been detected by ISOCAM (which is somewhat unrealistic since the ISOCAM detection limit at $z=0.75$ is $\sim 40 M_{\odot} \mathrm{yr}^{-1}$ ). Assuming the SFR derived from the IR luminosity for the ISOCAM-detected disks in L98, the average SFR at redshift $\sim 0.75$ would be on average $\sim 40 M_{\odot} \mathrm{yr}^{-1}$, or 6 times larger than the L98 average value. Further investigation will address the contribution of the massive galaxies to the CSFD (Hammer et al. 2004).

\subsection{Central color versus concentration index}

We have noticed that for most compact LIRGs (e.g. 03.0523, 03.0603, 03.0615 and 03.1540), the color maps have revealed a central region strikingly bluer than the outer regions. These blue central regions have a size similar to that of bulges and a color comparable to that of star-forming regions. Since the bulge/central region in local spirals is relatively red, such a blue-core structure could imply that the galaxy was (partially) forming the bulge. This is consistent with the scenario proposed by Hammer et al. (2001) that luminous compact galaxies are counterparts of the bulges in local spiral galaxies at intermediate redshifts. From color maps, the central regions (bulges?) in formation are revealed directly.

Let us assume that luminous compact galaxies are progenitors of the spiral cores/bulges and that disk components are formed later. Then a correlation is anticipated among star-forming systems (LIRGs: galaxies forming disks and/or bulges) between the color of their central region and the galaxy compactness. Star formation would first occur in the center (bulge) and would gradually migrate to the outskirts (disk), leading to redder colors of the central regions as the disk stars were forming. We have investigated the relation between the central color and the compactness for the LIRGs excluding the six major ongoing mergers, for which no central color is available due to their two separated components, and two objects whose structural parameters are not available. To reduce

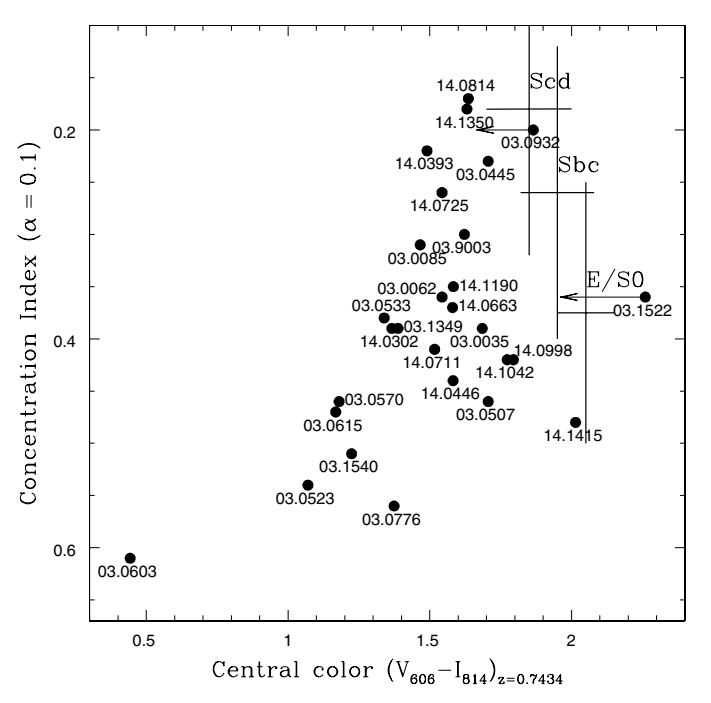

Fig. 13. Central color versus concentration index. The distributions (large crosses) of normal elliptical, Sab and Sbc galaxies are derived from a nearby galaxy sample (Frei et al. 1996). The modeling colors for the galaxies E, S0, Sbc and Irr at this redshift are 2.05, 1.88, 1.10 and 0.38 respectively (see Sect. 5.2.2). We assume that Sbc galaxies are bluer by $0.1 \mathrm{mag}$ than $\mathrm{E} / \mathrm{S} 0$ galaxies and Scd galaxies are $0.1 \mathrm{mag}$ bluer than Sbc galaxies in the bulge color (de Jong 1996). A central color of 2.05 is adopted for E/S0 galaxies. The CFRS ID is labeled for each data point. A Spearman rank-order (S-R) correlation analysis reveals a correlation coefficient of -0.42 with a probability of 0.026 that the null hypothesis of no correlation is true.

the contamination from the surrounding disk, a circle aperture with radius $1 \mathrm{kpc}$ centered on the $I_{814}$ band brightness peak is adopted to determine the central color. PHOT in IRAF $^{1}$ is used to do aperture photometry. This aperture includes 5 pixels for objects at redshift 1 and the random fluctuation is marginal due to the high $S / N$ in the central regions. We convert the observed central color $V_{606}-I_{814}$ or $B_{450}-I_{814}$ to the observed color $V_{606}-I_{814}$ at a median redshift of 0.7434 (see Hammer et al. 2001). At this reference redshift, The centroid wavelengths of the HST filters $I_{814}$ and $V_{606}$ correspond roughly to the $B$ band (4596 $\AA$ ) and $U$ band ( $3440 \AA$ ). The same method described in the previous subsection is used to estimate the $\mathrm{K}$-correction. We use the concentration index $(\alpha=0.1)$ defined in Abraham et al. (1994) to measure the compactness. It is provided as an output of GIM2D in the two-dimensional structure fitting. We also compute the same parameters for a population of local galaxies from Frei et al. (1996). The central color of the local galaxies has been assumed to be that of local ellipticals (for E and S0) or local bulges (for Sab to Sbc), and has been transformed to the $B(4596 \AA)-U(3440 \AA)$ color system using the GISSEL 98 model with $\tau=1$ Gyr. Figure 13 shows the investigation of the concentration index from the $I_{814}$ band as a function of the central color. In this diagram, the LIRGs are distributed along a sequence from galaxies with blue central color and compact morphology to galaxies with relatively red color and extended light distribution. Almost all distant LIRGs

\footnotetext{
${ }^{1}$ IRAF is distributed by the National Optical Astronomy Observatories, which are operated by the Association of Universities for Research in Astronomy, Inc. under cooperative agreement with the National Science Foundation.
} 
are discrepant from the sequence delineated by local spirals and ellipticals. There are two extreme cases, 03.0603 and 03.1522. The object 03.0603 could be contaminated by AGN because of its very blue point-like core. As mentioned in Sect. 6.1, the object 03.1522 is a very dusty edge-on spiral galaxy. Its very red central color is related to a (dust-screen) extinction. The sequence still exists when we replace the central color with the color contrast, which is defined as the difference between the central color and the integrated color ( $3^{\prime \prime}$ aperture).

The compact galaxies with a blue central color are suggested to be the galaxies forming their cores (bulges). Figure 13 clearly shows that these compact galaxies are located at one end of the sequence. The other end is occupied by extended galaxies with a concentration index and central color closer to those of local galaxies. It appears that such a sequence linking compact LIRGs to extended ones hints at a formation/assembly scenario for at least part of the present-day spiral galaxies: the bulge formed first and dominated a galaxy with a compact morphology and a relatively blue central color, and later the disk was assembled around the bulge, resulting in an extended light distribution which ultimately resembles those of local galaxies.

In trying to make sense of the sequence, we point out that intense star formation could happen in processes of both bulge and disk formation. In our color maps we see evidence that for LIRG spirals, star formation spreads over all their disks. A star formation history with several episodes of massive starbursts is suggested for LIRGs according to their composite stellar populations (Franceschini et al. 2003). A star formation history with multiple starburst episodes is also implied by the morphological classification of LIRGs such that massive starburst (luminous infrared phase) can happen in the spiral/irregular phase, major merger phase or compact phase. The major mergers in LIRGs would most likely result in spheroidal systems with violent starbursts, and become similar to the compact LIRGs with blue central colors such as those described in this paper. The spiral LIRGs could have undergone a compact stage before the observed epoch.

However, a simple evolutionary sequence does not account for all LIRGs. For example, among 12 LIRG disks with known bulge fraction, 3 are classified as $\mathrm{Sd}$, i.e. with a very small bulge, and 8 LIRGs are classified as irregulars. Examination of these $11 \mathrm{Sd}$ and Irr reveals the presence of a well-defined central component in most of them $(03.0085,03.0533,03.9003$, $14.0711,14.0725,14.0914,14.0998$ and 14.1190). On the other hand, such a component might not be a bulge (or a forming bulge), because it could be elongated or have peculiar shapes (14.0814 has an S-shape and it could be a giant bar). It is beyond the scope of this paper to evaluate the relative importance of each physical process (major and minor merging, bars, disk formation) that drive galaxy formation.

The scenario suggested by the sequence in Fig. 13 for disk galaxy formation is also supported by metallicity investigation of LIRGs by Liang et al. (2003). They found that, on average, the metal abundance of LIRGs is less than half that of the local disks with comparable brightness. They suggested that LIRGs form nearly half of their metals and stars since $z \sim 1$, assuming that they eventually evolve into the local massive disk galaxies.
It should be borne in mind that contribution from active galactic nuclei (AGNs) might strengthen this sequence since AGNs usually appear blue with respect to stellar populations (the situation is opposite if the AGNs are obscured severely by dust) and the existence of bright AGNs will bias the galaxies to the compact ones. However this concerns only a small fraction of our compact LIRGS (2 among 9). On the other hand, dust extinction will redden the color and smear the sequence.

\section{Conclusions}

Specific efforts were made to obtain the color maps for galaxies with complex morphology, including the accurate alignment of the blue and red band images and a new method to quantitatively determine the reliability of each pixel in the color map. These efforts allow us to access the spatially resolved color distribution of the distant LIRGs, which often have complex morphologies, relating to interactions/mergers. In two $10^{\prime} \times$ $10^{\prime}$ CFRS fields $0300+00$ and $1415+52$, HST WFPC2 imaging in $F 606 \mathrm{~W}$ (or $F 450 \mathrm{~W}$ ) and $F 814 \mathrm{~W}$ filters is available for an 87 square arcminute area. From these fields, we select a representative sample of 36 distant $(0.4<z<1.2)$ LIRGs detected in deep ISOCAM observations. A two-dimensional structure analysis was carried out using GIM2D software. With structure parameters and color distribution, a careful morphological classification was performed for the distant LIRGs. We find that about $36 \%$ LIRGs are spiral galaxies and about $25 \%$ LIRGs show compact morphology. About 22\% LIRGs are classified as irregular galaxies, showing complex and clumpy structures. Among 36 LIRGs, only $6(17 \%)$ of them were undergoing a major merger episode, revealed by a distinctive close galaxy pair with distorted morphology and apparent tidal tails. The fraction of mergers could reach $58 \%$ if all of the possible postmergers/pre-mergers are included.

Inspection of their stellar masses derived from $K$ band absolute magnitude shows that LIRGs are massive systems. The LIRGs classified as disk galaxies belong to the large disk galaxy population, and become a significant fraction of large distant disks selected by their sizes.

We find that LIRGs are distributed along a sequence in the central color versus compactness diagram. The sequence links the compact LIRGs with relatively blue central color to extended LIRGs with central color and compactness close to those of the local normal galaxies. The compact LIRGs showing blue central color are suggested to be systems forming their bulges, in agreement with the suggestion of Hammer et al. (2001). We argue that the sequence suggests that distant compact LIRGs would eventually evolve into spiral galaxies in the local universe.

Acknowledgements. We are grateful to Francoise Combes for helpful discussions and help in our morphological classification. We thank Jun Cui for his help in improving this manuscript. We thank the referee Dr. B. Mobasher for his helpful comments. X.Z.Z. gratefully acknowledges financial support from the Ministry of National Education of France. This work is based on observations made with the NASA/ESA Hubble Space Telescope, obtained from the data archive at the Space Telescope Institute. STScI is operated by the 
association of Universities for Research in Astronomy, Inc. under the NASA contract NAS 5-26555.

\section{References}

Abraham, R. G., Ellis, R. S., Fabian, A. C., Tanvir, N. R., \& Glazebrook, K. 1999, MNRAS, 303, 641

Abraham, R. G., Valdes, F., Yee, H. K. C., \& van den Bergh, S. 1994, ApJ, 432, 75

Berta, S., Fritz, J., Franceschini, A., Bressan, A., \& Lonsdale, C. 2004 [arXiv: astro-ph/0402211]

Bertin, E., \& Arnouts, S. 1996, A\&AS, 117, 393

Brinchmann, J., Abraham, R., Schade, D., et al. 1998, ApJ, 499, 112

Brinchmann, J., \& Ellis, R. S. 2000, ApJ, 536, L77

Bruzual, A. G., \& Charlot, S. 1993, ApJ, 405, 538

Conselice, C. J., Bershady, M. A., Dickinson, M., \& Papovich. C. 2003, AJ, 126, 1183

Crampton, D., Le Fèvre, O., Lilly, S. J., \& Hammer, F. 1995, ApJ, 455, 96

de Jong, R. S. 1996, A\&A, 313, 377

Dickinson, M., Papovich, C., Ferguson, H. C., \& Budavári, T. 2003, ApJ, 587, 25

Dolphin, A. E. 2000, PASP, 112, 1397

Dolphin, A. E. 2002, in 2002 HST Calibration Workshop: WFPC2 CTE Characterization, ed. S. Arribas, A. Koekemoer, \& B. Whitmore (Baltimore: STScI) 303

Elbaz, D., Cesarsky, C. J., Chanial, P., et al. 2002, A\&A, 384, 848

Ellis, R. S., Abraham, R. G., \& Dickinson, M. 2001, ApJ, 551, 111

Flores, H., Hammer, F., Thuan, T. X., et al. 1999, ApJ, 517, 148

Flores, H., et al. 2004, in preparation

Franceschini, A., Berta, S., Rigopoulou, D., et al. 2003, A\&A, 403, 501
Frei, Z., Guhathakurta, P., Gunn, J. E., \& Tyson, J. A. 1996, AJ, 111, 174

Genzel, R., \& Cesarsky, C. J. 2000, ARA\&A, 38, 761

Genzel, R., Tacconi, L. J., Rigopoulou, D., Lutz, D., \& Tecza, M. 2001, ApJ, 563, 527

Glazebrook, K., Peacock, J., Miller, L., \& Collins, C. 1995, MNRAS, 275,169

Hammer, F., Flores, H., Lilly, S. J., et al. 1997, ApJ, 481, 49

Hammer, F., Gruel, N., Thuan, T. X., Flores, H., \& Infante, L. 2001, ApJ, 550, 570

Hammer, F., Flores, H., Liang, Y. C., et al. 2004, in preparation

Groth, E. J., Kristian, J. A., Lynds, R., et al. 1994, A\&AS, 185, 5309

Koekemoer, A. M., Gonzaga, S., Fruchter, A., et al. 2002, HST Dither Handbook, Version 2.0 (Baltimore: STScI)

Le Fèvre, O., Abraham, R., Lilly, S. J., et al. 2000, MNRAS, 311, 565

Guzmán, R., Gallego, J., Koo, D. C., et al. 1997, ApJ, 489, 559

Lilly, S., Schade, D., Ellis, R., et al. 1998, ApJ, 500, 75

Lilly, S., Le Fèvre, O., Crampton, D., Hammer, F., \& Tresse, L. 1995, ApJ, 455, 50

Liang, Y. C., Hammer, F., Flores, H., Elbaz, D., \& Cesarsky, C. J. 2003, A\&A, submitted

Madau, P., Ferguson, H. C., Dickinson, M. E., et al. 1996, MNRAS, 283, 1388

Menanteau, F., Abraham, R. G., \& Ellis, R. S. 2001, MNRAS, 322, 1

Rola, C., \& Pelat, D. 1994, A\&A, 287, 676

Schade, D., Lilly, S. J., Crampton, D., et al. 1999, ApJ, 525, 31

Simard, L., Willmer, C. N. A., Vogt, N. P., et al. 2002, ApJS, 142, 1

Trauger, J. T., Vaughan, A. H., Evans, R. W., \& Moody, D. C. 1995, in Calibrating Hubble Space Telescope: Post Servicing Mission, ed. A. Koratkar, \& C. Leitherer (Baltimore: STScI), 379

Williams, R. E., Blacker, B., Dickinson, M., et al. 1996, AJ, 112, 1335 


\section{Online Material}


X. Z. Zheng et al.: Distant LIRGs - morphologies and color maps, Online Material $p 2$

Table 1. HST imaging with two band observations in CFRS fields $0300+00$ and 1415+52.

\begin{tabular}{ccccccccccc}
\hline \hline Field & Blue filter & Total Exp. & $N^{a}$ & Dither $^{b}$ & Red filter & Total Exp. & $N^{a}$ & Dither $^{b}$ Prop. ID $^{c}$ \\
\hline $030226+001348$ & $F 606 W$ & 6400 & 5 & 20 & $F 814 W$ & 6000 & 5 & 20 & 9149 \\
$030227+000704$ & $F 450 W$ & 7000 & 5 & 20 & $F 814 W$ & 6700 & 5 & 20 & 6556,5996 \\
$030233+001255$ & $F 450 W$ & 6600 & 6 & 0 & $F 814 W$ & 6400 & 6 & 0 & 5449 \\
$030237+001414$ & $F 606 W$ & 6400 & 5 & 20 & $F 814 W$ & 6400 & 5 & 20 & 9149 \\
$030240+000940$ & $F 606 W$ & 6400 & 5 & 20 & $F 814 W$ & 7000 & 5 & 12.5 & 9149,8162 \\
$030243+001324$ & $F 450 W$ & 6600 & 6 & 0 & $F 814 W$ & 6400 & 6 & 0 & 5449 \\
$030250+001000$ & $F 606 W$ & 6400 & 5 & 20 & $F 814 W$ & 7000 & 5 & 12.5 & 9149,8162 \\
$141743+523025$ & $F 450 W$ & 7800 & 6 & 0 & $F 814 W$ & 7400 & 6 & 0 & 5449 \\
$141803+522755$ & $F 606 W$ & 6400 & 5 & 20 & $F 814 W$ & 6800 & 5 & 20 & 9149 \\
$141809+523015$ & $F 450 W$ & 7800 & 6 & 0 & $F 814 W$ & 7400 & 6 & 0 & 5449 \\
$141724+522512$ & $F 606 W$ & 2800 & 4 & 0 & $F 814 W$ & 4400 & 4 & 0 & 5090 \\
$141731+522622$ & $F 606 W$ & 2800 & 4 & 0 & $F 814 W$ & 4400 & 4 & 0 & 5090 \\
$141737+522731$ & $F 606 W$ & 2800 & 4 & 0 & $F 814 W$ & 4400 & 4 & 0 & 5090 \\
$141750+522951$ & $F 606 W$ & 2800 & 4 & 0 & $F 814 W$ & 4400 & 4 & 0 & 5090 \\
$141743+522841$ & $F 606 W$ & 24400 & 12 & 0 & $F 814 W$ & 25200 & 12 & 0 & 5109 \\
$141757+523101$ & $F 606 W$ & 2800 & 4 & 0 & $F 814 W$ & 4400 & 4 & 0 & 5090 \\
$141803+523211$ & $F 606 W$ & 2800 & 4 & 0 & $F 814 W$ & 4400 & 4 & 0 & 5090 \\
\hline
\end{tabular}

${ }^{a}$ Number of exposures.

${ }^{b}$ Dither offset among the consecutive exposures, aimed to remove cosmic-rays and hot/bad pixels. Here the largest offset in unit of pixel along $x$ axis is present.

${ }^{c}$ HST Proposal ID. One ID means that both the blue and the red observations were carried out in the same proposal. Two IDs refer to the proposals that the blue and the red observations were observed respectively. 
X. Z. Zheng et al.: Distant LIRGs - morphologies and color maps, Online Material p 3

Table 2. Offsets and rotation between blue and red images.

\begin{tabular}{|c|c|c|c|}
\hline Field & $\Delta x$ (pixel) & $\Delta y$ (pixel) & $\Delta \theta\left(^{\circ}\right)$ \\
\hline \multicolumn{4}{|c|}{ CFRS fields } \\
\hline $030226+001348$ & -2.28 & 2.50 & 0.550 \\
\hline $030227+000704$ & 4.31 & -14.00 & 0.188 \\
\hline $030233+001255$ & 0.00 & -0.15 & 0.000 \\
\hline $030237+001414$ & 5.54 & -5.37 & -1.200 \\
\hline $030240+000940$ & 9.76 & 8.90 & -0.185 \\
\hline $030243+001324$ & -0.05 & -0.36 & 0.000 \\
\hline $030250+001000$ & 20.65 & 7.04 & 0.070 \\
\hline $141743+523025$ & 0.35 & -0.32 & 0.000 \\
\hline $141803+522755$ & -6.47 & 7.15 & 1.600 \\
\hline $141809+523015$ & 0.03 & -0.36 & 0.000 \\
\hline $221755+001715$ & -8.59 & -3.99 & 0.188 \\
\hline \multicolumn{4}{|c|}{ Groth strip fields } \\
\hline $141527+520410$ & 0.25 & 0.05 & 0.000 \\
\hline $141534+520520$ & 0.19 & 0.08 & 0.000 \\
\hline $141540+520631$ & 0.19 & 0.13 & 0.000 \\
\hline $141547+520741$ & 0.18 & 0.05 & 0.000 \\
\hline $141553+520851$ & 0.18 & 0.08 & 0.000 \\
\hline $141600+521001$ & 0.29 & 0.08 & 0.000 \\
\hline $141606+521111$ & 0.17 & 0.07 & 0.000 \\
\hline $141613+521222$ & 0.19 & 0.05 & 0.000 \\
\hline $141619+521332$ & 0.21 & 0.04 & 0.000 \\
\hline $141626+521442$ & 0.23 & 0.17 & 0.000 \\
\hline $141632+521552$ & 0.26 & 0.13 & 0.000 \\
\hline $141638+521702$ & 0.22 & 0.10 & 0.000 \\
\hline $141645+521812$ & 0.31 & 0.10 & 0.000 \\
\hline $141651+521922$ & 0.31 & 0.03 & 0.000 \\
\hline $141658+522032$ & 0.19 & 0.07 & 0.000 \\
\hline $141704+522142$ & 0.10 & 0.10 & 0.000 \\
\hline $141711+522252$ & 0.20 & 0.11 & 0.000 \\
\hline $141717+522402$ & 0.15 & 0.02 & 0.000 \\
\hline $141724+522512$ & 0.21 & 0.08 & 0.000 \\
\hline $141731+522622$ & 0.20 & -0.01 & 0.000 \\
\hline $141737+522731$ & 0.20 & 0.03 & 0.000 \\
\hline $141750+522951$ & -0.02 & 0.11 & 0.000 \\
\hline $141757+523101$ & 0.21 & 0.09 & 0.000 \\
\hline $141803+523211$ & 0.25 & 0.02 & 0.000 \\
\hline $141810+523320$ & 0.27 & 0.08 & 0.000 \\
\hline $141816+523430$ & 0.26 & 0.05 & 0.000 \\
\hline $141823+523540$ & 0.09 & 0.06 & 0.000 \\
\hline
\end{tabular}

\section{Appendix: Signal-to-noise ratio for color map}

A color image is a difference of two images in a logarithm function (here we ignore the scaling constant which will not affect the final results). Given one HST image in the blue band with

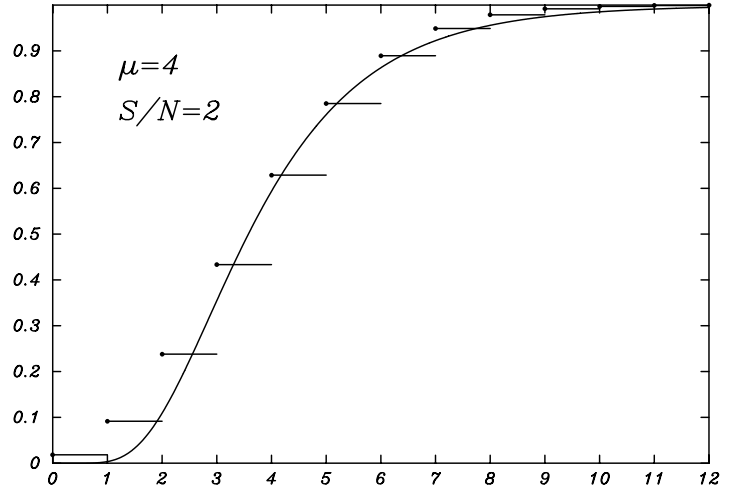

Fig. A.1. The distribution function of Poisson noise at $S / N=2$ and its Log-normal approximation (continuous line) for the same $S / N$ ratio. Even at this low $S / N$ the two functions differ by a value of 0.15 at most.

flux $F_{B}$ and another one in the red band with flux $F_{R}$, the color image is defined as

$Y=\log F_{B}-\log F_{R}$

Here, the flux is a function of the position in an image, including signals of background and object. The HST images have Poisson noise. We know the signals $\mu_{F_{B}}, \mu_{F_{R}}$ and the noises $\sigma_{F_{B}}^{2}, \sigma_{F_{R}}^{2}$ in the two images, respectively. We intend to derive signal $\mu$ and noise $\sigma^{2}$ of the color image and then obtain its $S / N$ ratio. The issue with color representation of an image is to avoid the divergence at zero implied by the logarithm function. A way to do this is to replace the Poisson noise by a random variable possessing zero probability at zero value. This approximation must be valid at low $S / N$ ratio, say $S / N \geq 2$. It is clear that such an approximation must break down at very low $S / N$, say $S / N \leq 1$ where the Poisson random variable is zero most of the time. Here we adopt an approximation that the Poisson noise distribution function in the HST image is close to a Lognormal law. A similar approach was tested in a spectroscopic context by Rola \& Pelat (1994). It is shown in Fig. A.1 that even at $S / N=2$ a Log-normal variable is an acceptable approximation of a Poisson one.

In logarithms, the blue image $B=\log F_{B}$ and the red image $R=\log F_{R}$ satisfy a Normal law. A difference between two Normal law images is also a Normal law image. This property is the main advantage of using the log-normal law to approximate the Poisson noise. The signal $\mu$ and noise $\sigma^{2}$ of the color image are given by

$\mu=\mu_{B-R}=\mu_{B}-\mu_{R}$

and

$\sigma^{2}=\sigma_{B-R}^{2}=\sigma_{B}^{2}+\sigma_{R}^{2}$

where $\mu_{B}$ and $\sigma_{B}^{2}, \mu_{R}$ and $\sigma_{R}^{2}$ are the signal and the noise of the Normal law images $B$ and $R$ respectively. For log-normal function $\exp Y$, its signal $m$ and noise $s^{2}$ can be written as

$m=\exp \left(\mu+\frac{1}{2} \sigma^{2}\right)$ 
Table 3. Catalog of the deep ISOCAM-detected sources in CFRS fields $1300+00$ and $1415+52$.

\begin{tabular}{|c|c|c|c|c|c|c|c|c|c|c|c|c|c|c|c|c|}
\hline \multirow[b]{2}{*}{$\begin{array}{l}\text { CFRS ID } \\
\text { (1) }\end{array}$} & \multirow[b]{2}{*}{$\begin{array}{c}z \\
(2)\end{array}$} & \multirow[b]{2}{*}{$\begin{array}{l}m_{450} \\
(3)\end{array}$} & \multirow[b]{2}{*}{$\begin{array}{c}m_{606} \\
(4)\end{array}$} & \multirow[b]{2}{*}{$\begin{array}{c}m_{814} \\
(5)\end{array}$} & \multirow[b]{2}{*}{$\begin{array}{c}M_{\mathrm{AB}}(B) \\
(6)\end{array}$} & \multirow[b]{2}{*}{$\begin{array}{c}M_{\mathrm{AB}}(K) \\
\quad(7)\end{array}$} & \multirow[b]{2}{*}{$\begin{array}{c}L_{\mathrm{IR}}\left(10^{10} L_{\odot}\right) \\
\quad(8)\end{array}$} & \multicolumn{2}{|c|}{$\overline{-B_{450}}$} & \multicolumn{2}{|c|}{$V_{606}$} & \multicolumn{2}{|c|}{$I_{814}$} & \multirow[b]{2}{*}{$\begin{array}{l}\text { Type }^{a} \\
(15)\end{array}$} & \multirow[b]{2}{*}{$\begin{array}{l}Q^{b} \\
(16)\end{array}$} & \multirow[b]{2}{*}{$\begin{array}{l}\text { Int } / M^{c} \\
(17)\end{array}$} \\
\hline & & & & & & & & $\begin{array}{l}B / T \\
(9)\end{array}$ & $\begin{array}{c}\chi^{2} \\
(10)\end{array}$ & $\begin{array}{l}B / T \\
(11)\end{array}$ & $\begin{array}{l}\chi^{2} \\
(12)\end{array}$ & $\begin{array}{l}B / T \\
(13)\end{array}$ & $\begin{array}{l}\chi^{2} \\
(14)\end{array}$ & & & \\
\hline \multicolumn{17}{|c|}{ No redshift identification } \\
\hline 03.0028 & - & - & 21.16 & 19.30 & - & - & - & - & - & $0.71_{-0.02}^{+0.01}$ & 1.078 & $0.73_{-0.01}^{+0.01}$ & 1.047 & & & \\
\hline 03.0115 & - & - & 21.15 & 19.53 & - & - & - & - & - & $0.13_{-0.01}^{+0.02}$ & 1.588 & $0.22_{-0.01}^{+0.01}$ & 1.474 & & & \\
\hline 03.0121 & - & - & 21.40 & 19.76 & - & - & - & - & - & $0.20_{-0.07}^{+0.01}$ & 1.947 & $0.18_{-0.02}^{+0.01}$ & 1.413 & & & \\
\hline 03.0346 & - & 23.77 & - & 21.52 & - & - & - & $0.72_{-0.06}^{+0.10}$ & 1.089 & -0.07 & - & $0.43_{-0.01}^{+0.02}$ & 2.375 & & & \\
\hline 14.0324 & - & - & 22.85 & 21.47 & - & - & - & & - & $0.27_{-0.05}^{+0.13}$ & 1.0156 & $0.38_{-0.05}^{+0.01}$ & 1.0075 & & & \\
\hline 14.0405 & - & - & 22.26 & 20.38 & - & - & - & & - & $\begin{array}{l}-0.05 \\
0.36_{-0.04}^{+0.06}\end{array}$ & 1.0004 & $0.19_{-0.02}^{+0.05}$ & 1.0183 & & & \\
\hline \multicolumn{17}{|c|}{$z \leq 0.4$} \\
\hline 03.0355 & 0.0870 & 19.06 & - & 16.91 & -19.31 & - & $1.52 \pm 0.32$ & $0.34_{+0.02}^{-0.02}$ & 1.183 & - & - & $0.70_{+0.00}^{-0.01}$ & 1.383 & & & \\
\hline 03.0364 & 0.2513 & 20.97 & - & 18.95 & -20.30 & -22.14 & $4.56 \pm 0.81$ & $0.00_{+0.01}^{-0.02}$ & 1.520 & - & - & $0.03_{+0.00}^{+0.00}$ & 1.787 & & & \\
\hline 03.0365 & 0.2187 & 21.10 & - & 18.89 & -19.57 & -21.68 & $4.26 \pm 0.87$ & $0.18_{+0.02}^{+0.02}$ & 1.374 & - & - & $0.00_{+0.00}^{-0.00}$ & 1.420 & & & \\
\hline 03.0443 & 0.1178 & 20.37 & - & 19.12 & -19.15 & - & $0.78 \pm 0.16$ & $0.72_{+0.05}^{+0.02}$ & 0.952 & - & - & $0.70_{+0.02}^{+0.00}$ & 1.093 & & & \\
\hline 03.0495 & 0.2614 & - & 20.38 & 19.25 & -19.82 & - & $5.38 \pm 1.22$ & - & - & $0.32_{+0.01}^{-0.01}$ & 2.174 & $0.40_{+0.07}^{+0.02}$ & 1.906 & & & \\
\hline 03.0569 & 0.1810 & - & 22.41 & 21.27 & -16.73 & -20.01 & $1.67 \pm 0.37$ & - & - & - & - & - & - & & & \\
\hline 03.0578 & 0.2200 & - & 21.59 & 20.93 & -18.64 & -19.52 & $1.58 \pm 0.36$ & - & - & $0.13_{+0.02}^{-0.01}$ & 1.084 & $0.21_{+0.05}^{-0.05}$ & 0.989 & & & \\
\hline 03.0711 & 0.2620 & - & 21.34 & 20.51 & -18.55 & -20.44 & $2.51 \pm 0.58$ & - & - & $0.00_{+0.00}^{+0.02}$ & 1.298 & $0.00_{+0.00}^{+0.05}$ & 1.136 & & & \\
\hline 03.0949 & 0.0330 & 19.40 & - & 17.56 & -16.86 & - & $0.18 \pm 0.02$ & $0.24_{+0.03}^{-0.08}$ & 0.897 & $\begin{array}{l}0.000 \\
-\end{array}$ & - & $0.13_{+0.12}^{+0.00}$ & 1.225 & & & \\
\hline 03.1299 & 0.1760 & - & 19.66 & 18.76 & -19.94 & - & $5.02 \pm 1.23$ & $\begin{array}{c}0.4+0.03 \\
-\end{array}$ & - & $0.13_{+0.02}^{-0.02}$ & 3.688 & $0.10_{+0.07}^{-0.12}$ & 1.005 & & & \\
\hline 03.1311 & 0.1760 & - & 20.30 & 19.36 & -18.86 & -21.69 & $2.09 \pm 0.49$ & - & - & $0.00_{+0.00}^{+0.02}$ & 1.562 & $0.04_{+0.01}^{+0.07}$ & 1.327 & & & \\
\hline 14.0435 & 0.0684 & - & 18.71 & 17.91 & -18.30 & - & $0.14 \pm 0.02$ & - & - & $0.00_{+0.00}^{+0.00}$ & 1.481 & $0.00_{+0.00}^{+0.01}$ & 1.395 & & & \\
\hline 14.1257 & 0.2927 & - & 21.24 & 20.16 & -19.44 & -22.45 & $15.97 \pm 2.15$ & - & - & $0.00_{+0.00}^{+0.00}$ & 1.859 & $0.00_{+0.00}^{+0.00}$ & 1.490 & & & \\
\hline 14.1329 & 0.3750 & - & 20.21 & 19.19 & -21.00 & - & $13.03 \pm 2.47$ & - & - & $0.43_{+0.02}^{-0.00}$ & 1.976 & $0.48_{+0.01}^{-0.00}$ & 1.816 & & & \\
\hline 14.9025 & 0.1550 & - & 19.57 & 18.69 & -19.77 & - & $1.69 \pm 0.39$ & - & - & $0.00_{+0.00}^{+0.02}$ & 3.280 & $0.07_{+0.00}^{+0.01}$ & 2.186 & & & \\
\hline \multicolumn{17}{|c|}{$0.4<z<1.2$} \\
\hline 03.0035 & 0.8804 & 24.06 & 22.69 & 21.12 & -21.87 & -23.70 & $171.47 \pm 23.41$ & - & - & $0.28_{+0.06}^{-0.08}$ & 1.349 & $0.31_{+0.04}^{-0.06}$ & 0.993 & $\mathrm{Sab}(3.6)$ & 2 & $\mathrm{R}$ ? \\
\hline 03.0062 & 0.8252 & - & 22.26 & 20.90 & -21.84 & -23.00 & $111.73 \pm 13.74$ & - & - & $0.00_{+0.00}^{+0.00}$ & 1.545 & $0.01_{+0.01}^{+0.04}$ & 1.439 & Sbc (3.0) & 1 & \\
\hline 03.0085 & 0.6100 & - & 22.58 & 21.51 & -19.98 & -21.09 & $29.55 \pm 2.67$ & - & - & $0.00_{+0.01}^{+0.00}$ & 1.274 & - & - & $\mathrm{Sd}(5.1)$ & 2 & \\
\hline 03.0445 & 0.5300 & 22.84 & - & 20.40 & -21.25 & - & $21.28 \pm 4.27$ & $1.00^{-0.03}$ & 0.992 & + & - & $0.01^{-0.00}$ & 1.038 & Sbc (3.9) & 2 & $\mathrm{R}$ ? \\
\hline 03.0507 & 0.4660 & - & 21.33 & 20.48 & -20.47 & -21.23 & $11.57 \pm 2.71$ & - & - & $0.07_{+0.012}^{-0.01}$ & 1.325 & $0.24_{+0.08}^{+0.00}$ & 1.227 & $\mathrm{Sab}(3.2)$ & 2 & \\
\hline 03.0523 & 0.6508 & 23.03 & 22.00 & 20.99 & -20.93 & -21.73 & $68.65 \pm 4.36$ & $0.34_{+0.55}^{-0.29}$ & 0.931 & $0.53_{+0.04}^{+0.12}$ & 1.789 & $0.42_{+0.05}^{+0.08}$ & 1.292 & $\mathrm{C} / \mathrm{T}$ & 1 & M 2 \\
\hline 03.0533 & 0.8290 & - & 22.30 & 21.02 & -21.38 & -22.28 & $170.04 \pm 23.67$ & $\begin{array}{l}+0.55 \\
-\end{array}$ & - & $0.00_{+0.00}^{+0.04}$ & 1.096 & $0.00_{+0.00}^{-0.05}$ & 1.170 & Irr & 1 & $\mathrm{R}$ \\
\hline 03.0570 & 0.6480 & - & 22.67 & 21.69 & -20.12 & -20.58 & $29.97 \pm 2.75$ & - & - & $0.03^{-0.003}$ & 1.242 & $0.42_{+0.06}^{+0.00}$ & 1.111 & $\mathrm{C}$ & 1 & \\
\hline 03.0603 & 1.0480 & - & 21.26 & 20.37 & -23.01 & -23.71 & $435.58 \pm 83.86$ & - & - & $0.43_{+0.01}^{+0.05}$ & 1.464 & $0.73_{+0.01}^{+0.06}$ & 1.290 & $\mathrm{C}$ & 1 & $\mathrm{R}$ \\
\hline 03.0615 & 1.0480 & - & 22.52 & 21.51 & -21.66 & -22.77 & $344.69 \pm 62.39$ & - & - & $0.35_{+0.04}^{+0.01}$ & 1.390 & $0.33_{+0.02}^{+0.01}$ & 1.306 & $\mathrm{C}$ & 3 & \\
\hline 03.0776 & 0.8830 & - & 23.28 & 22.05 & -20.69 & -20.98 & $107.84 \pm 14.25$ & - & - & $0.00_{+0.08}^{+0.04}$ & 0.992 & $0.04_{+0.09}^{+0.02}$ & 1.061 & $\mathrm{C}$ & 1 & \\
\hline $03.0916^{d}$ & 1.0300 & 22.64 & - & 21.17 & -22.23 & - & $503.26 \pm 68.64$ & - & - & $\begin{array}{l}+0.08 \\
-\end{array}$ & - & -0 & - & $\mathrm{C}$ & 1 & \\
\hline 03.0932 & 0.6478 & 23.54 & - & 21.18 & -20.73 & -22.85 & $97.18 \pm 6.95$ & - & - & - & - & $0.00_{+0.01}^{-0.00}$ & 1.046 & Sbc (7.0) & 1 & \\
\hline 03.1309 & 0.6170 & - & 21.65 & 20.77 & -21.49 & -23.00 & $89.28 \pm 6.32$ & - & - & - & - & 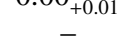 & - & & 4 & M 1 \\
\hline
\end{tabular}


Table 3. continued.

\begin{tabular}{|c|c|c|c|c|c|c|c|c|c|c|c|c|c|c|c|c|}
\hline \multirow[b]{2}{*}{$\begin{array}{c}\text { CFRS ID } \\
\text { (1) }\end{array}$} & \multirow[b]{2}{*}{$\begin{array}{c}z \\
(2)\end{array}$} & \multirow[b]{2}{*}{$\begin{array}{l}m_{450} \\
(3)\end{array}$} & \multirow[b]{2}{*}{$\begin{array}{l}m_{606} \\
(4)\end{array}$} & \multirow[b]{2}{*}{$\begin{array}{c}m_{814} \\
(5)\end{array}$} & \multirow[b]{2}{*}{$\begin{array}{c}M_{\mathrm{AB}}(B) \\
\quad(6)\end{array}$} & \multirow[b]{2}{*}{$\begin{array}{c}M_{\mathrm{AB}}(K) \\
\quad(7)\end{array}$} & \multirow[b]{2}{*}{$\begin{array}{c}L_{\mathrm{IR}}\left(10^{10} L_{\odot}\right) \\
\quad(8)\end{array}$} & \multicolumn{2}{|c|}{$B_{450}$} & \multicolumn{2}{|c|}{$-V_{606}$} & \multicolumn{2}{|c|}{$I_{814}$} & \multirow[b]{2}{*}{$\begin{array}{l}\text { Type }^{a} \\
\text { (15) }\end{array}$} & \multirow[b]{2}{*}{$\begin{array}{l}Q^{b} \\
(16)\end{array}$} & \multirow[b]{2}{*}{$\begin{array}{l}\text { Int/M } \mathbf{M}^{c} \\
(17)\end{array}$} \\
\hline & & & & & & & & $\begin{array}{r}B / T \\
(9) \\
\end{array}$ & $\begin{array}{c}\chi^{2} \\
(10)\end{array}$ & $\begin{array}{l}B / T \\
(11)\end{array}$ & $\begin{array}{c}\chi^{2} \\
(12)\end{array}$ & $\begin{array}{l}B / T \\
(13)\end{array}$ & $\begin{array}{c}\chi^{2} \\
(14)\end{array}$ & & & \\
\hline 03.1349 & 0.6155 & - & 21.67 & 20.38 & -21.15 & -22.63 & $56.77 \pm 3.38$ & - & - & $0.28_{+0.01}^{-0.01}$ & 1.179 & $0.39_{+0.08}^{-0.09}$ & 0.873 & $\mathrm{Sab}(4.5)$ & 1 & $\mathrm{I} 2$ \\
\hline 03.1522 & 0.5870 & 25.73 & - & 21.74 & -19.59 & -21.96 & $46.13 \pm 3.02$ & - & - & - & - & $0.22_{+0.28}^{-0.16}$ & 0.994 & $S(9.0)$ & 1 & \\
\hline 03.1540 & 0.6898 & 22.95 & - & 20.61 & -21.27 & - & $90.23 \pm 9.19$ & $0.64_{+0.36}^{-0.64}$ & 0.932 & - & - & $0.52_{+0.06}^{-0.04}$ & 1.223 & $\mathrm{C}$ & 1 & $\mathrm{R} ?$ \\
\hline 03.9003 & 0.6189 & 22.58 & - & 20.47 & -21.24 & - & $54.74 \pm 3.73$ & $0.13_{+0.04}^{-0.02}$ & 1.863 & - & - & $0.00_{+0.00}^{-0.00}$ & 2.017 & Irr & 1 & M 2 \\
\hline 14.0302 & 0.5830 & 22.78 & - & 20.44 & -20.98 & -22.70 & $56.94 \pm 3.17$ & $0.28_{+0.16}^{-0.18}$ & 1.005 & - & - & $0.22_{+0.04}^{-0.03}$ & 1.069 & Sab (3.0) & 2 & $\mathrm{R} ?$ \\
\hline 14.0393 & 0.6016 & 23.07 & - & 20.94 & -21.64 & -22.30 & $55.15 \pm 3.57$ & - & - & - & - & $0.04_{+0.01}^{-0.04}$ & 1.157 & Sbc (3.6) & 1 & \\
\hline 14.0446 & 0.6030 & - & 21.15 & 19.88 & -21.95 & - & $56.87 \pm 3.29$ & - & - & $0.00_{+0.00}^{-0.00}$ & 1.243 & $0.00_{+0.00}^{-0.00}$ & 1.363 & $\mathrm{Sd}(6.8)$ & 1 & \\
\hline 14.0547 & 1.1963 & - & 22.02 & 21.17 & -23.12 & -23.88 & $186.03 \pm 43.71$ & - & - & - & - & - & - & & 4 & M 1 \\
\hline $14.0600^{e}$ & 1.0385 & - & 22.03 & 21.13 & -22.04 & - & $369.49 \pm 61.12$ & - & - & - & - & - & - & Irr & 1 & \\
\hline 14.0663 & 0.7434 & - & 21.98 & 20.51 & -21.65 & - & $148.43 \pm 18.98$ & - & - & $0.02_{+0.01}^{-0.01}$ & 1.241 & $0.15_{+0.01}^{-0.01}$ & 1.155 & Sbc (3.1) & 2 & \\
\hline 14.0711 & 1.1180 & - & 22.30 & 21.24 & -22.50 & -23.18 & $463.42 \pm 96.59$ & - & - & $0.42_{+0.06}^{-0.16}$ & 1.166 & $0.03_{+0.05}^{-0.03}$ & 1.228 & Irr & 1 & M 2 \\
\hline 14.0725 & 0.5820 & - & 22.86 & 21.66 & -19.47 & -21.26 & $24.12 \pm 3.27$ & - & - & $0.00_{+0.01}^{-0.00}$ & 1.027 & $0.00_{+0.00}^{-0.00}$ & 1.091 & Irr & 3 & \\
\hline 14.0814 & 0.9995 & - & 23.56 & 22.04 & -20.73 & -22.02 & $163.02 \pm 84.25$ & - & - & $0.01_{+0.02}^{-0.01}$ & 1.076 & $0.00_{+0.01}^{-0.00}$ & 1.159 & Irr & 3 & $\mathrm{R} ?$ \\
\hline 14.0998 & 0.4300 & 23.02 & 21.27 & 20.09 & -20.32 & -22.17 & $26.08 \pm 4.24$ & $0.08_{+0.32}^{-0.08}$ & 0.867 & $0.00_{+0.00}^{-0.02}$ & 1.891 & $0.00_{+0.00}^{-0.00}$ & 1.582 & Irr & 3 & M 2 \\
\hline 14.1042 & 0.8916 & - & 22.75 & 20.98 & -21.60 & -23.07 & $99.37 \pm 10.51$ & $0.21_{+0.28}^{-0.21}$ & 1.008 & $0.01_{+0.00}^{-0.01}$ & 1.189 & $0.31_{+0.01}^{-0.01}$ & 1.352 & $\mathrm{C}$ & 1 & M 2 \\
\hline 14.1129 & 0.8443 & - & 22.59 & 21.36 & -21.86 & -22.81 & $123.68 \pm 15.89$ & - & - & - & - & - & - & & 4 & M 1 \\
\hline 14.1139 & 0.6600 & 22.40 & 21.20 & 20.24 & -22.02 & -23.47 & $118.00 \pm 11.36$ & - & - & - & - & - & - & & 4 & M 1 \\
\hline 14.1157 & 1.0106 & - & 22.44 & 19.95 & -23.11 & - & $1786^{f}$ & - & - & - & - & - & - & & 4 & M 1 \\
\hline 14.1190 & 0.7544 & - & 21.97 & 20.57 & -21.58 & - & $92.43 \pm 9.42$ & - & - & $0.02_{+0.01}^{-0.01}$ & 1.309 & $0.03_{+0.00}^{-0.00}$ & 1.373 & $\mathrm{Sd}(2.6)$ & 2 & $\mathrm{R} ?$ \\
\hline 14.1305 & 0.8069 & - & 23.29 & 21.59 & -20.73 & - & $67.30 \pm 7.23$ & - & - & - & - & - & - & & 4 & M 1 \\
\hline 14.1350 & 1.0054 & - & 22.70 & 21.43 & -21.44 & - & $297.35 \pm 139.92$ & - & - & $0.01_{+0.01}^{-0.01}$ & 1.085 & $0.00_{+0.00}^{-0.00}$ & 1.171 & Irr & 2 & \\
\hline 14.1415 & 0.7486 & - & 22.47 & 20.67 & -21.48 & - & $87.44 \pm 9.98$ & - & - & $0.23_{+0.03}^{-0.02}$ & 1.076 & $0.42_{+0.01}^{-0.02}$ & 1.142 & $\mathrm{C}$ & 2 & $\mathrm{R}$ \\
\hline
\end{tabular}

${ }^{d}$ This object is too compact to obtain structural parameters.

${ }^{e}$ Structural parameters are not successfully derived for this object from the two-dimensional structure fitting because of its clumpy light distribution and imagery close to the CCD chip border.

${ }^{f}$ Infrared luminosity is estimated according to an obscured AGN model.

Notes: Structural parameters are not provided for the merging systems with multiple distinctly separated components. 
X. Z. Zheng et al.: Distant LIRGs - morphologies and color maps, Online Material p 6

and

$s^{2}=\left(\exp \sigma^{2}-1\right) \exp \left(2 \mu+\sigma^{2}\right)$.

Expressing $\mu$ and $\sigma^{2}$ in $m$ and $s^{2}$, we obtain

$\mu=-\frac{1}{2} \log \left(\frac{s^{2}}{m^{2}}+1\right)+\log m$

and

$\sigma^{2}=\log \left(\frac{s^{2}}{m^{2}}+1\right)$
With $\exp Y=\frac{F_{B}}{F_{B}}$, we obtain the signal and noise for the color

(6) $\mu=-\frac{1}{2} \log \left(\frac{\sigma_{F_{B}}^{2}}{\mu_{F_{B}}}+1\right)+\log \mu_{F_{B}}+\frac{1}{2} \log \left(\frac{\sigma_{F_{R}}^{2}}{\mu_{F_{R}}}+1\right)-\log \mu_{F_{R}}(9)$

(7) and $\sigma^{2}=\log \left(\frac{\sigma_{F_{B}}^{2}}{\mu_{F_{B}}^{2}}+1\right)+\log \left(\frac{\sigma_{F_{R}}^{2}}{\mu_{F_{R}}^{2}}+1\right)$, 